\title{
Fusing Vision and Inertial Sensors for Robust Runway Detection and Tracking
}

\author{
Khaled Abu-Jbara* and Ganesh Sundaramorthi \pm \\ KAUST, Thuwal 23955-6900, Saudi Arabia \\ and \\ Christian Claudel \\ University of Texas, Austin, Texas \\ DOI: $10.2514 / 1 . G 002898$
}

\begin{abstract}
This work presents a novel real-time algorithm for runway detection and tracking applied to unmanned aerial vehicles (UAVs). The algorithm relies on a combination of segmentation-based region competition and minimization of a particular energy function to detect and identify the runway edges from streaming video data. The resulting video-based runway position estimates can be updated using a Kalman filter (KF) that integrates additional kinematic estimates such as position and attitude angles, derived from video, inertial measurement unit data, or positioning data. This allows a more robust tracking of the runway under turbulence. The performance of the proposed lane detection and tracking scheme is illustrated on various experimental UAV flights conducted by the Saudi Aerospace Research Center (KACST), by the University of Texas, Austin, and on simulated landing videos obtained from a flight simulator. Results show an accurate tracking of the runway edges during the landing phase, under various lighting conditions, even in the presence of roads, taxiways, and other obstacles. This suggests that the positional estimates derived from the video data can significantly improve the guidance of the UAV during takeoff and landing phases.
\end{abstract}

$\begin{array}{ll} & \text { Nomenclature } \\ E & =\text { energy function } \\ \Omega & =\text { domain of the image } \\ \theta & =\text { angle of line segment, rad } \\ \nabla & =\text { gradient }\end{array}$

\section{Introduction}

$\mathbf{U}$ NMANNED aerial vehicles (UAVs) have become increasingly prevalent in the past decade, enabling monitoring tasks that are too dirty, too dull, or too dangerous (DDD) to be undertaken by manned flying vehicles. UAVs have, for instance, been used in surveillance applications, including fire detection [1] , event detection [2], or object tracking [3]. They also play an increasing role in military operations [4] for both reconnaissance and strike. While rotorcraft UAVs are most commonly used, fixed-wing UAVs have in general a higher autonomy and endurance, owing to their relatively high lift-to-drag ratio, and are thus preferred for certain types of operations (e.g., the surveillance of a target, or search and rescue operations). However, takeoff and landings of fixed-wing UAVs are particularly risky [5], particularly because lightweight UAVs are much more sensitive to turbulence than heavier manned aircraft, and thus have much lower time constants. In currently operated UAVs, takeoffs and landings are typically carried out using navigation sensors, such as absolute positioning systems (e.g., Global Positioning System [GPS]), accelerometers, gyrometers, and magnetometers. While the fusion between these sensors greatly improves their accuracy, the residual positional errors are still too large to allow reliable UAV landings on narrow UAV airstrips. Positioning accuracy on practical UAV airstrips could be improved by using enhanced GPS systems (such as differential GPS, or RTK GPS), though such systems require additional

Received 13 March 2017; revision received 8 February 2018; accepted for publication 11 March 2018; published online Open Access XX epubMonth XXXX. Copyright (C) 2018 by the American Institute of Aeronautics and Astronautics, Inc. All rights reserved. All requests for copying and permission to reprint should be submitted to CCC at www.copyright.com; employ the ISSN 0731-5090 (print) or 1533-3884 (online) to initiate your request. See also AIAA Rights and Permissions www.aiaa.org/randp.

*Ph.D. Candidate, Department of Electrical Engineering.

${ }^{\dagger}$ Assistant Professor, Department of Electrical Engineering.

${ }^{\sharp}$ Assistant Professor, Department of Civil, Architectural and Environmental Engineering, Austin 301 E. Dean Keeton St. Stop C1761. equipment (reference stations, phase sensing antennas), which is expensive. The use of high-accuracy positioning systems also require the UAV airstrips to be precisely mapped, though this positioning information is most of the time unavailable. In contrast, the ever decreasing cost of cameras makes them particularly suitable to this positioning application. Because UAVs are mainly used for surveillance applications, the vast majority of UAV flights are conducted during good weather conditions, during which the visibility is high. To help the guidance of UAVs during takeoff and landing phases, we propose a new algorithm leveraging image processing and control systems theory to detect the runway edges. The resulting position estimates can then be used to more accurately correct the lateral position of the UAV during the landing phase, and use higher gains in the UAV control loop, resulting in better tracking performance during the takeoff and landing phases. The main objective of the proposed algorithm is to build a very robust detection and tracking of the runway edges, which performs well under diverse lighting and environmental conditions. Because video data of UAV landings are scarce, supervised learning would yield poor performance. Numerous computer vision algorithms have been proposed to help the navigation of fixed-wing and rotary UAVs. In [6] , the authors use morphological image processing and HTs to identify the horizon and estimate the attitude of a UAV. The authors of [7] propose a template matching algorithm to detect and track the position of a runway. Active methods can also be thought of, for instance, in [8], which uses active infrared emitters to guide the UAV during its landing. In other contexts, computer vision has been successfully used to detect road lanes (for autonomous vehicle applications), for instance, in [9] , where roads are detected using HT and Robert filters. The authors of [10] use splines curve fitting, HT, Canny edge detector, and vanishing points to estimate the boundary of the road. Although effective, this method is complex and does not run in real time on commercially available embedded platforms. In paper [11] a line segment detector (LSD) is used to detect the main line features, and K-means clustering is applied to sort and select specific lines. The authors of [4] have a different approach, relying on a Sobel filter and K-means to find the edges of the road from a fixed surveillance camera. Other approaches for lane or road detection exist, such as in [12], in which the authors use Random Sample Consensus (RANSAC), and cubic spline curve fitting are used to extract the lane position from a video stream. A linear parabolic lane tracking system with 
Kalman filter (KF) is proposed in [13]. On the other hand, many approaches, such as $[14,15]$, tend to use convex methods in PDE optimization to segment the image into $N$ regions based on global intensity statistics or local intensity statistics.

To the best of the authors knowledge, no robust runway detection and tracking scheme capable of working under a wide variety of lighting conditions and a variety of runway configurations (including the presence of obstacles and taxiways) is currently available. Currently available methods, such as [16], require the user to specify in advance a very large number of parameters associated with the current tracking problem or to specify in advance which features (for instance, landmarks or shadows [10]) have to be excluded from the tracking problem. These requirements significantly complicate operations and require recalibration before each flight and before changing the takeoff and landing locations. The present algorithm uses a minimal number of assumptions on the features associated with all runways and relies on robust video detection and tracking methods, which offer sufficient performance in practice to be used standalone. In the present paper, we also investigate a $\mathrm{KF}$ approach that can fuse additional information (such as gyrometer data) in the prediction step, to enhance tracking accuracy.

We illustrate this problem of sensitivity to model parameters on Fig. 1, which shows the difficulty of robustly selecting the contours of the image that are relevant to the proposed problem. This figure illustrates the considerable modification of the output of a Canny edge detector (applied to a UAV landing video) caused by minor changes in its parameters. The upper left subfigure shows the actual video data, whereas the upper right, lower left, and lower right subfigures correspond to the outputs of the Canny edge detector applied to this image, using the parameters $\sigma=1$ (upper right), $\sigma=10$ (lower left), and $\sigma=20$ (lower right), in which $\sigma$ denotes to the standard deviation of the Gaussian Filter, with upper and lower thresholds of 0.4 and 0.1 , respectively. As can be seen from these images, a small variation of the parameters can dramatically affect the features extracted from the image. Recent edge detectors [17] exhibit a similar sensitivity to parameters.

The rest of the paper is organized as follows. Section II.A gives a high-level overview of the proposed runway detection and tracking algorithm. The core vision-based feature extraction algorithm is described in Sec. II.B, and formulated as an optimization problem. Section III introduces the KF formulation of the runway edge detection and tracking algorithm. We validate in Sec. IV the performance of this algorithm using experimental landing videos obtained by the Saudi Department of Aerospace and from the University of Texas, Austin. In this section, we show that runway
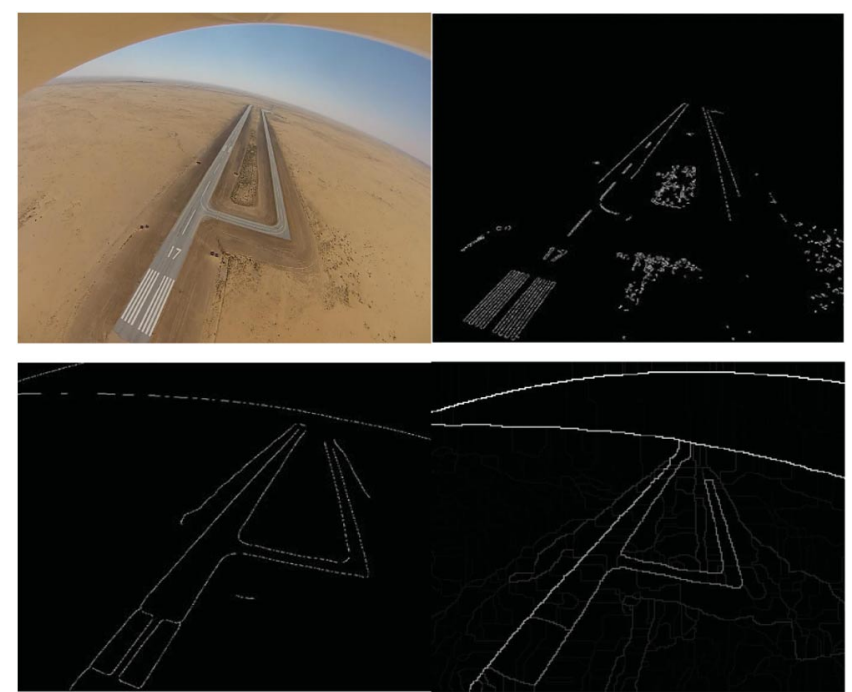

Fig. 1 Canny edge detection results on experimental landing video image. detection and tracking is achieved in a very wide variety of initial conditions and lighting conditions, without any modification of the parameters of the algorithm.

\section{Runway Detection and Tracking Algorithm Structure}

In this section, we formulate a visual model of runways in aerial images. We then use the model to formulate an optimization problem to detect and segment the runway on a given image, based on the minimization of an energy function. Our key innovations are a model and optimization methods that are specifically designed to lead to efficient computational algorithms that can be implemented on embedded platforms from off-the-shelf UAVs. Furthermore, the algorithms are designed to be robust to the background clutter, illumination conditions (e.g., day and night), and noise. These features are prevalent in aerial imagery obtained during the takeoff and landing phases and make the detection and tracking of a runway significantly more complex.

\section{A. Model and Optimization Problem}

We model the runway as two line segments (one for each edge of the runway). Typical runways are flat enough to be approximated as two parallel straight lines, which due to perspective projection may appear nonparallel in the image. Although runways may consist of multiple intersecting runways, in which case the two-line-segment assumption may fail, we wish to detect the runway localized to a certain region around the longitudinal axis of the aircraft, where the two-line-segment assumption is largely true. Such a model is simple and robust, which makes the image processing faster and more reliable. We also assume that the image intensity statistics are maximally different in a neighborhood of each of the edges delimiting the runway. This translates the fact that the color and intensity associated with the runway is very different from the color and intensity associated with the neighborhood of the runway. Figure $\underline{3}$ illustrates this example, in the case of a runway located in a desert. This is the main assumption used by the proposed algorithm and is true in most settings. We make no assumption to the rest of the image, such as the background and the interior of the runway, as runways can have very different features, including ground markings, or the presence of taxiways.

For simplicity, we assume that the aircraft is approximately aligned with the runway at the initial time. This assumes that either the pilot flies toward the runway or that the autopilot flies the UAV in the general direction of the runway. This hypothesis is not restrictive in practice because commercially available GPS and inertial sensors have a positioning uncertainty of less than a few tens of meters (typically less than $10 \mathrm{~m}$ for the GPS), and uncertainty in heading measurement of fewer than $10 \mathrm{deg}$. In this case, within a rectangle around the runway, we may assume that both line segments representing the runway intersect the top and bottom of the rectangle. The rectangle corresponds to the approximate localization of the runway within the image and can be initialized with a guess of the approximate position of the runway, obtained, for example, using the navigation system of the UAV, from approximate coordinates of the runway.

The line segments representing the runway on the image can thus be described by four coordinates $d_{i}, i=1, \ldots, 4$, representing the distance between the corners of the rectangle and the endpoints of the line segments, as illustrated in Fig. 3 .

We now formulate an optimization problem to determine the values of the coordinates $d_{i}, i=1, \ldots, 4$ that best represent the current position of the runway, within the image. As noted earlier, the runway is such that the neighboring regions on either side of the line segments have maximally differing image intensity statistics. For simplicity, we assume that the statistics are the mean RGB values with the neighborhoods, but any other statistics could be used, such as intensity histograms, statistics of filter bank responses, or statistics on a specific color hue (e.g., if the runway is known to be in a grassy, snowy, or desert environment). Let the image be denoted by $I: \Omega \rightarrow \boldsymbol{R}^{k}$ (with $k=3$ for RGB color encoding), where $\Omega \subset \boldsymbol{R}^{2}$, 


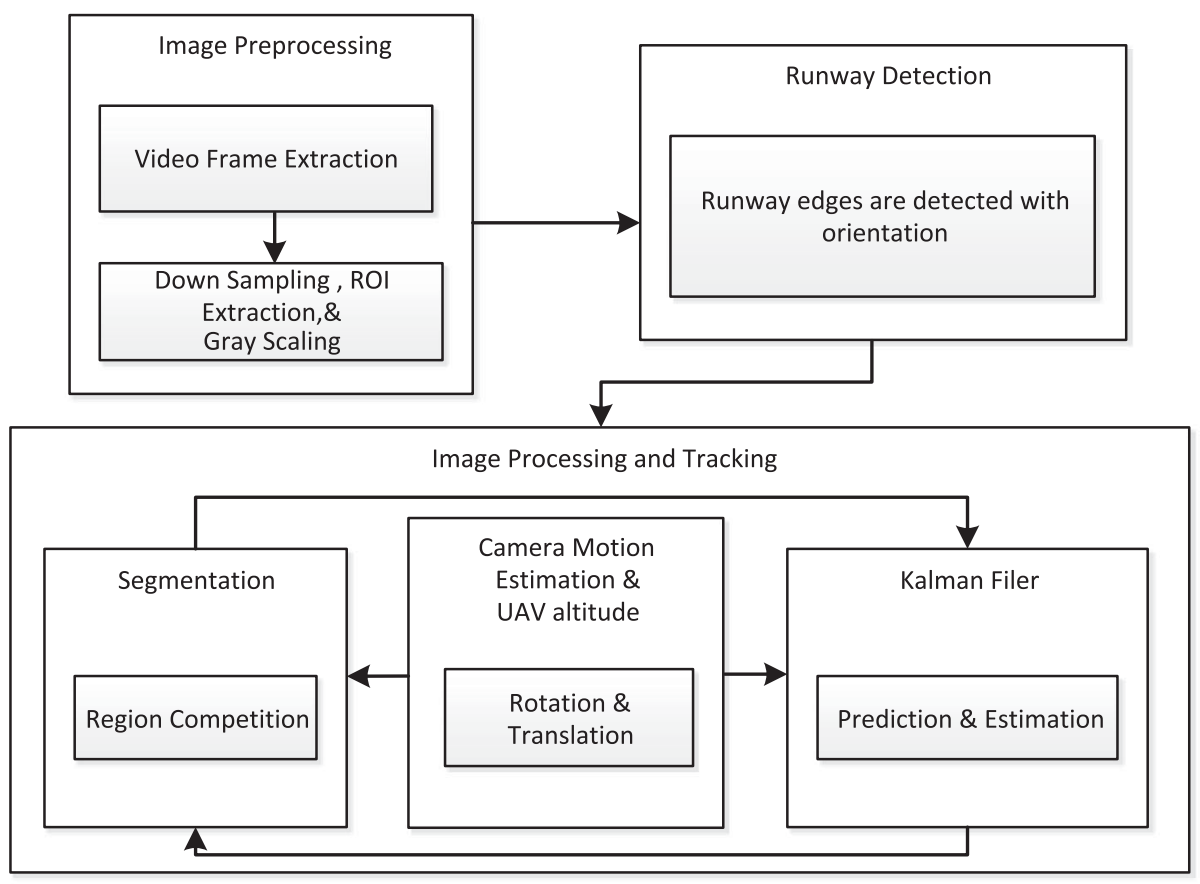

Fig. 2 Overview of the runway detection and tracking algorithm developed in this paper.

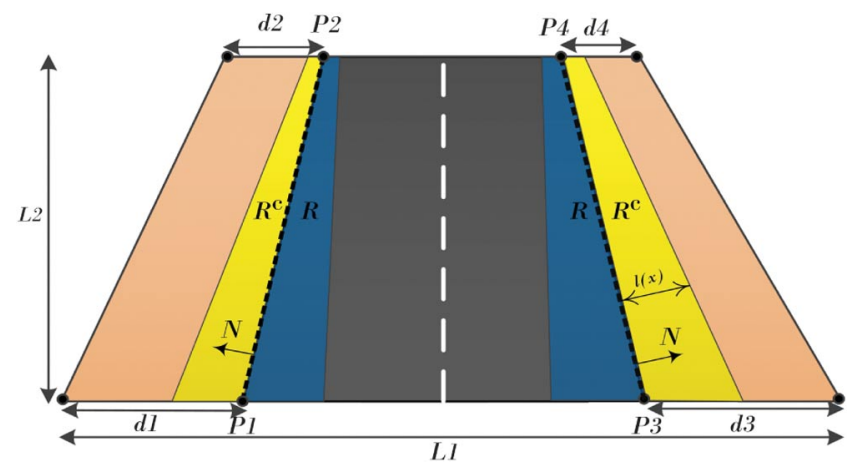

Fig. 3 Runway model considering the perspective projection using measurements of the orientation and position of the camera.

and let $R, R^{c} \subset \Omega$ denote the inside and outside neighborhood regions of the line segments, respectively, as in Fig. 3. The mean statistics $u, v \in \boldsymbol{R}^{k}$ for the inner and outer neighborhoods defined at a certain point $x$ on the line segment become

$$
\begin{aligned}
& u(x)=\frac{1}{\ell(x)} \int_{0}^{\ell(x)} I(x-s N) \mathrm{d} s, \\
& v(x)=\frac{1}{\ell(x)} \int_{0}^{\ell(x)} I(x+s N) \mathrm{d} s
\end{aligned}
$$

where $N$ is the unit outward normal to the line segment corresponding to either side (right or left) of the trapezoid, $d s$ is the arclength differential element of the segment defined on $[x, x-\ell(x) N]$, $[x, x+\ell(x) N]$ for $u, v$ respectively, and we would define that as the spatially variant mean calculation.

As a result of the perspective projection from three-dimensional (3D) to two-dimensional (2D), $\ell(x)$ would be a function of $x$. Consequently, this would cause the trapezoidal neighborhood around the line segments using measurements of the orientation and position of the camera. Refer to Sec. III.B for more details on $\ell(x)$.
For the special case, having $R, R^{c}$ nonspatially variant, the mean statistics expression is simplified to

$$
u=\frac{1}{|R|} \int_{R} I(x) \mathrm{d} A, \quad v=\frac{1}{\left|R^{c}\right|} \int_{R^{c}} I(x) \mathrm{d} A
$$

where $|R|$ denotes the area of $R$, and $d A$ is an area element.

Because we would like to define the optimal line segments such that the two statistics, $u$ and $v$, are maximally different; our objective is to minimize the following energy function:

$$
E\left(d_{1}, d_{2}, d_{3}, d_{4}\right)=-\frac{1}{2} \int_{\partial R}|u(x)-v(x)|^{2} \mathrm{~d} s(x)+\gamma \sum_{i=1}^{4}\left|d_{i}-d_{i}^{\prime}\right|^{2}
$$

where $\partial R$ is the boundary of $R$ that is allowed to vary, and $d s(x)$ is the arclength differential element of the segment defined on $\left[0, L_{2}\right]$.

Note that $u$ and $v$ are functions of the position of the line segments because $R$ and $R^{c}$ depend on the line segments, which are in turn specified by $d_{i}$. Therefore, the energy $E$ itself is only a function of the coordinates $d_{i}, i=1, \ldots, 4$.

Because the image is not expected to move too much between frames, we add a regularization term to penalize abrupt changes of the coordinates $d_{i}, i=1, \ldots, 4$ from the initial guess denoted by $d_{i}^{\prime}$, $i=1, \ldots, 4$. This prevents oversegmentation if the region around the runway includes outliers. Higher values of $\gamma$ will limit the freedom of the lines' displacement toward convergence and vice versa. For practical implementations, $\gamma$ can be set a priori, or adaptive, depending on the amount of clutter in the neighborhood of the runway.

As will be shown later in the paper, the use of region statistics in the design of the energy leads to a robust runway detection algorithm. Local derivatives, which are widely used in edge detection, are sensitive to noise and extraneous features in the images. Thus, using statistics that are more robust to such disturbances will lead to an algorithm that is less sensitive to irrelevant image features. The larger the neighborhoods, $R$ and $R^{c}$, the more robust the statistics are to local disturbances. However, larger neighborhoods encompass more of the background where our assumptions of maximally differing statistics 
of $u$ and $v$ may not hold due to irrelevant clutter. This tradeoff in size is important and will be analyzed in the subsequent sections.

The energy above is related to a large body of literature in image segmentation by partial differential equations-based methods [18-20] in which the energies are defined on the set of (infinite dimensional) regions. The energy is most closely related to [21], where the objective is to divide the image into two disjoint regions that have maximally different intensity means. Our energy formulation has two major improvements with respect to previously developed formulations:

1) The energy is defined on a simplified representation of regions defined by line segments to support real-time applications needed for UAV landing.

2) We rely on neighborhoods rather than the entire image to avoid background clutter typical in aerial imagery.

One problem with image segmentation methods based on partial differential equations is that they usually rely on local gradient descent methods, because the energy function is nonconvex (as is our energy of interest). Therefore, they require the user to initialize the algorithm properly so that the algorithm converges toward the global minimum of the energy function. Our model of the runway is designed to achieve a computationally tractable detection algorithm that localizes the runway in real time on standard embedded computers.
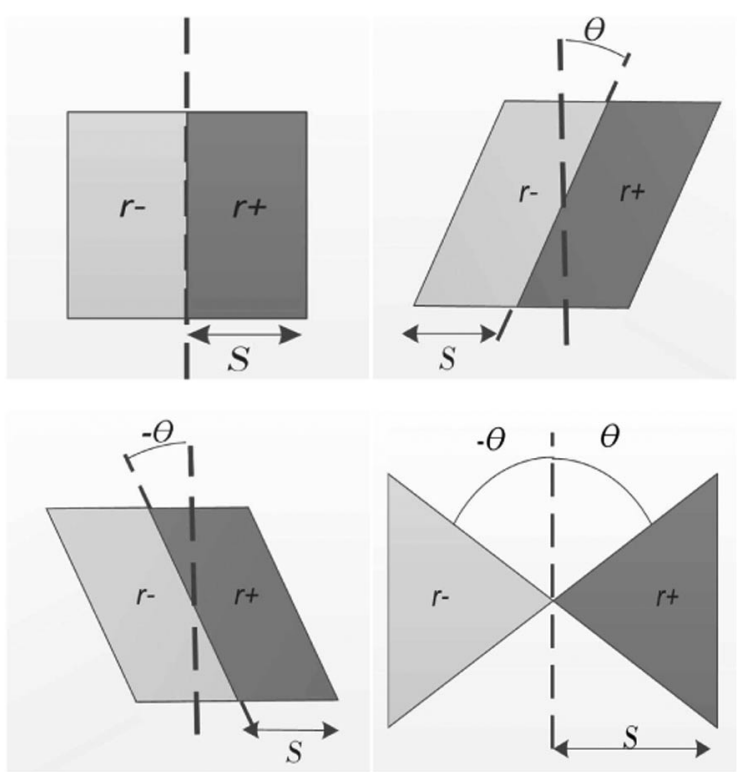

Fig. 4 Edge detectors for various orientations. The lower right region is the intersection of all $r+$ and $r_{-}$within a specified range $\left[-\theta_{m}, \theta_{m}\right]$; it is able to detect lines with any orientation without searching within the range.

\section{B. Detection Method}

The evaluation of $E\left(d_{1}, \ldots, d_{4}\right)$ for every $\left(d_{1}, \ldots, d_{4}\right)$ on a four-dimensional computational grid would be computationally prohibitive and too slow for real-time runway tracking applications. To minimize the number of candidate grid points, we need to have a good initial guess of the position of the runway. In the present application, we cannot assume that the runway is approximately located initially, because its coordinates may be unavailable, or position sensors may be malfunctioning (e.g., in GPS-denied environments). Therefore, local optimization techniques, which are the only candidates for optimization of $E$ due to nonconvexity, could fail to initially detect the runway and possibly lead to crash if the runway position information is used in the control loop. To address this issue, we construct an algorithm that efficiently searches per the set of candidates $\left(d_{1}, \ldots, d_{4}\right)$. The algorithm prunes away irrelevant line segments in this set and proposes a few candidate segments as potential initialization points. The energy of these few candidates can then be evaluated, and the energetically most favorable candidate can be selected. This allows us to detect the runway immediately, without any convergence period, and without initializing the algorithm with the estimated initial position of the runway.

To identify candidate pairs of line segments outlining the runway, we first focus on generating candidate single line segments separating the runway with the rest of the image. Each line segment is described by two coordinates: $\theta(\theta \in[-\pi / 2, \pi / 2])$, which corresponds to the angle of the line segment with respect to the vertical axis, and the horizontal position $x$ of the center of the line segment. The present algorithm efficiently computes minimizers of

$$
E^{\prime}(x, \theta)=-\frac{1}{2}(\bar{u}(x, \theta)-\bar{v}(x, \theta))^{2}
$$

where $\bar{u}$ and $\bar{v}$ are the mean values of the chosen statistics on either side of the segment described by $x, \theta$.

Evaluating $E^{\prime}$ for all $x, \theta$ on a $2 \mathrm{D}$ computational grid would be too slow for real-time applications. Therefore, we introduce a hierarchical search, to first determine candidate locations $x$, and then determine the second coordinate $\theta$. For a fixed $x$, a diagram illustrating the neighborhoods (called $r_{+}(\theta), r_{-}(\theta)$ ) of the line segments corresponding to different orientations $\theta$ is shown in Fig. 4 .

Let us now consider the intersections $r_{+, \text {inter }}=\cap_{\theta \in \mathcal{D}} r_{+}(\theta)$ and $r_{-, \text {inter }}=\cap_{\theta \in \mathcal{D}} r_{-}(\theta)$ of the neighborhoods $r_{+}$and $r_{-}$for $\theta$ ranging in $\mathcal{D}$. These intersected regions are shown in the bottom right in Fig. 4. If the difference between $r_{+, \text {inter }}$ and $r_{-, \text {inter }}$ is small, then the coordinate $x$ is not a good candidate location for a runway edge. Such locations are thus eliminated from the search, allowing us to quickly determine candidate locations $x$. We define the response $\mathcal{R}$ as $\left(r_{+, \text {inter }}-r_{-, \text {inter }}\right)^{2}$. The response is only a function of the coordinate $x$. An example of response function is shown in Fig. 5. The left subfigure shows the response function of the image, associated with coordinates centered between the two horizontal green lines of the center subfigure, whereas the right subfigure depicts the determination of the correct candidate coordinates $x_{i}, \theta_{i}$.
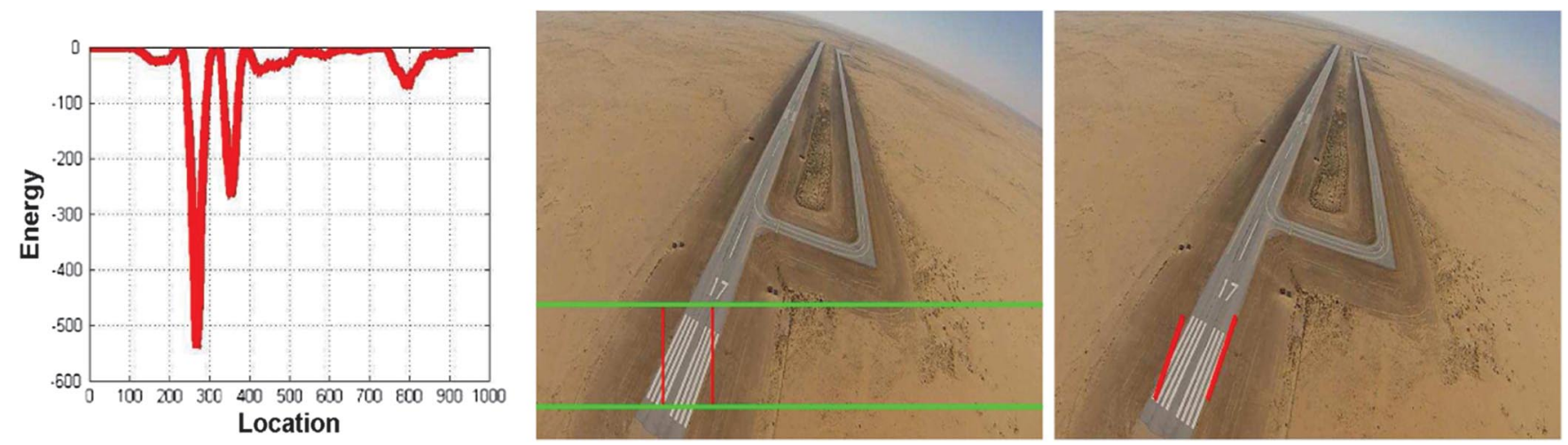

Fig. 5 Energy profile and candidate line segments outlining the runway. 


\section{Algorithm 1: Runway detection.}

1) For each $x \in\left\{0,1, \ldots, L_{1}-1\right\}$

a) compute $u$ and $v$ with regions defined by the mask in Fig. $\underline{4}$ (bottom, right) and centered at $\left(x, L_{2} / 2\right)$ for $\theta=\pi / 4$

b) compute $\mathcal{R}(x)=-(u-v)^{2} / 2$

2) compute the $N$ lowest local minima of $\mathcal{R}$, called $x_{i}, i=1, \ldots, N$

$3)$ compute the $\theta \in\{-\pi / 4,-\pi / 8,0, \pi / 8, \pi / 4\}$ corresponding to $x_{i}$ that minimizes $E$ for $i=1, \ldots, N$

4) compute $E$ for all pairs of line segments determined by $\left(x_{i}, \theta_{i}\right)$

5) output the pair of line segments $\left(x_{i}, y_{i}\right),\left(x_{j}, y_{j}\right)$ that have minimum $E$

The best candidate locations of line segments corresponding to edges of the runway are local maxima of $\mathcal{R}$, that is, the locations associated with the highest responses. Because $r_{+, \text {inter }}$ has fewer pixels than any particular $r_{+}$, the means $r_{+, \text {inter }}$ and $r_{- \text {,inter }}$ are more susceptible to noise, and therefore computing local minima may generate false positives. We eliminate these false positives by doing a search over a coarse grid $\theta \in\{-\pi / 4,-\pi / 8,0, \pi / 8, \pi / 4\}$, only at the candidate local maxima locations, and retain only candidate locations that minimize the energy function. The actual orientation is then determined by performing a search on a fine grid. This procedure gives candidate coordinates $\left(x_{i}, \theta_{i}\right), i=1, \ldots, N$ for line segments of the runway, where $N$ corresponds to the number of candidates retained by the algorithm. In subsequent implementations we choose $N=5$.

Once we have obtained the candidate line segments, we compute the energy $E$ for pairs of line segments in the candidate list $\left(x_{i}, \theta_{i}\right)$. The pair with the lowest energy is selected as the detected runway, after running $N(N-1)$ energy computations. Because $N$ is small, this procedure is considerably faster than evaluating the energy of all possible pairs of line segments, corresponding to a search over a four-dimensional grid.

The algorithm used for the runway detection procedure is summarized in Algorithm 1.

To link the image processing in $2 \mathrm{D}$ and the data fusing in $3 \mathrm{D}$, a rough location estimate in $3 \mathrm{D}$ is given via GPS (runway is in the vicinity). Hence, the $2 \mathrm{D}$ image processing-lane detection starts to work, only by sweeping horizontally, between the green lines in lower part of the image where originally the runway starts to appear. This distance between the green lines is related to the elevation of the UAV above the ground; with lower altitude it increases. It can be roughly estimated based on observations from landing videos, and then to be saved as a look-up table.

\section{Local Optimization for Refined Segmentation}

Once a candidate runway has been identified, we run a local optimization method to minimize E on an image, given an initialization close to the desired runway. We choose a local optimization approach for multiple reasons:

1) The detection procedure outlined in the previous section only gives us a coarse approximation of the position and orientation of with respect to the UAV, because it is obtained by performing a search over a relatively coarse computational grid.

2) Assuming that the runway was correctly localized on the previous frame, we can process the current frame with a very good initial guess (assuming that the frame rate of the camera is sufficient, and the dynamics of the UAV are sufficiently slow). Therefore, a local optimization is sufficient to solve the problem, and allows both a higher precision and a faster computational time than a search over the entire image.

3) A local optimization approach allows us to regularize the solutions with respect to time, if multiple runway candidates are possible (e.g., if in the presence of multiple parallel runways or taxiways).

Local optimization is relatively fast, and less computationally costly than running the global detection procedure outlined in the previous section. Therefore, a gradient descent method $[22,23]$ is used to minimize $E$. One may interpret the energy $E$ as defined on a closed contour, that is, a trapezoid formed by the line segments. This description allows us to use results from the partial differential equation (PDE) image segmentation literature [18-20], which have derived methods for optimizing arbitrary energies defined on closed contours that are allowed to deform arbitrarily. However, in optimizing $E$, we would like to enforce the constraint that the trapezoidal shape of the runway section remains a trapezoid on the subsequent frame. This type of constraint can be enforced by restricting the possible contour deformations to trapezoid preserving deformations [24].

The gradient of the cost function is the sum of two terms, one term associated with the energy $E:(\nabla E)_{i}=\left(\partial E / \partial d_{i}\right)_{i}$, for $i \in\{1, \ldots, 4\}$ (this term can be computed as line integrals, as shown in Proposition 1), and a second term, $2 \gamma\left(d_{i}-d_{i}^{\prime}\right)$, associated with the regularization term of the objective function.

The gradient descent algorithm thus optimizes the parameters $d$ using a recursive equation

$$
d_{k+1}=d_{k}-\Delta d \nabla E\left(d_{k}\right)
$$

where $d_{k}$ are the coordinates computed at step $k$. We initialize $d_{0}$ with the coordinates of the runway associated with the previous frame, and choose an adaptive step size $\Delta d=0.5 /\left\|\nabla E\left(d_{k}\right)\right\|$ to speed up convergence.

Proposition 1: The gradient of $E$ is given by $\nabla E=\left(\partial E / \partial d_{i}\right)_{i=1}^{4}$, where the partial derivatives are given by:

$$
\begin{aligned}
\frac{\partial E}{\partial d_{1}} & =L_{2}(v-u)\left[\frac{1}{|R|} \int_{0}^{1}(t-1)\left(I\left(C_{L_{1}}(t)\right)-u_{L_{1}}(t)\right) \mathrm{d} t\right. \\
& \left.+\frac{1}{\left|R^{c}\right|} \int_{0}^{1}(t-1)\left(I\left(C_{L_{1}}(t)\right)-v_{L_{1}}(t)\right) \mathrm{d} t\right]
\end{aligned}
$$

$$
\begin{aligned}
& \frac{\partial E}{\partial d_{2}}=L_{2}(v-u)\left[\frac{1}{|R|} \int_{0}^{1} t\left(I\left(C_{L_{1}}(t)\right)-u_{L_{1}}(t)\right) \mathrm{d} t\right. \\
& \left.\quad+\frac{1}{\left|R^{c}\right|} \int_{0}^{1} t\left(I\left(C_{L_{1}}(t)\right)-v_{L_{1}}(t)\right) \mathrm{d} t\right]
\end{aligned}
$$

$$
\begin{aligned}
\frac{\partial E}{\partial d_{3}} & =L_{2}(v-u)\left[\frac{1}{|R|} \int_{0}^{1}(t-1)\left(I\left(C_{L_{2}}(t)\right)-u_{L_{2}}(t)\right) \mathrm{d} t\right. \\
+ & \left.\frac{1}{\left|R^{c}\right|} \int_{0}^{1}(t-1) I\left(\left(C_{L_{2}}(t)\right)-v_{L_{1}}(t)\right) \mathrm{d} t\right] \\
\frac{\partial E}{\partial d_{4}} & =L_{2}(v-u)\left[\frac{1}{|R|} \int_{0}^{1} t\left(I\left(C_{L_{2}}(t)\right)-u_{L_{2}}(t)\right) \mathrm{d} t\right. \\
& \left.+\frac{1}{\left|R^{c}\right|} \int_{0}^{1} t\left(I\left(C_{L_{2}}(t)\right)-v_{L_{2}}(t)\right) \mathrm{d} t\right]
\end{aligned}
$$

$$
u_{L_{i}}(t)=\frac{1}{\ell(t)} \int_{0}^{\ell(t)} I\left(C_{L_{i}}(t)-s \cdot N\right) \mathrm{d} s
$$




$$
v_{L_{i}}(t)=\frac{1}{\ell(t)} \int_{0}^{\ell(t)} I\left(C_{L_{i}}(t)+s \cdot N\right) \mathrm{d} s
$$

where $C_{L_{i}}$ is the arc-parameterization of the $i$ th line segment corresponding to $\left(d_{1}, \ldots, d_{4}\right) ; L_{2}$ is the image width; $u$ and $v$ are the means inside the regions $R$ and $R^{c}$, respectively; $N_{i}$ is the unit outward normal to $C_{L_{i}}$; and $d s$ is the arclength differential element of the segment defined on $\left[C_{L_{i}}(t), C_{L_{i}}(t)-\ell(t) N\right],\left[C_{L_{i}}(t), C_{L_{i}}(t)+\ell(t) N\right]$, for $u_{L_{i}}(t), v_{L_{i}}(t)$, respectively.

\section{Tracking}

\section{A. Dynamical Model of the Camera}

Because virtually all UAVs are equipped with positioning and inertial systems, we can leverage additional data generated by the IMU and positioning systems of the UAV to better initialize the gradient descent algorithm in highly turbulent conditions, or when the camera has a low frame rate (e.g., in poor lighting conditions). In earlier work [25], we assumed that the apparent velocity of the runway was approximately constant. This approximation holds only if the attitude of the UAV does not change too abruptly over time. In this paper, we fuse the inertial and positional data generated by the UAV sensors to better estimate the position of the runway on a new frame, allowing a faster convergence of the gradient descent method outlined in the previous section. Because our dynamic model is the runway position in the $3 \mathrm{D}$ world coordinates of a reference frame, the method arising from the model also allows one to estimate the relative position between the UAV and the runway, which is important in closed-loop operation, when the relative position is fed into a controller of the UAV.

We now describe our representation for the runway, which then allows us to define its dynamic model. Instead of representing the runway as four sets of coordinates (positions of the start and end points of the segments limiting the runway), we assume that the runway is described by two infinite parallel lines. We also enforce geometrical constraints on the runway that the runway can be described by two parallel lines in the Earth frame. These two previous assumptions allow us to reduce the dimensionality of the problem, with only four independent coordinates instead of eight:

$$
\left\{\begin{array}{ll}
a x+b y+c=0, & \text { Line } 1 \\
a x+b y+d=0, & \text { Line } 2
\end{array}\right\}
$$

where $(x, y)$ describe points on the ground $(z=0)$ on the lines, and $a, b, c, d \in \boldsymbol{R}$ are parameters of the lines. Because a given line of equation $a x+b y+c=0$ can be equivalently described as $\alpha a x+\alpha b y+\alpha c=0$ for nonzero $\alpha$, we further impose

$$
\sqrt{a^{2}+b^{2}}=1
$$

to enforce uniqueness.

Our dynamical model for the runway is simply that the runway remains stationary with respect to the world frame, which implies that $a, b, c, d$ remain constant across time. To fuse all sources of information (camera, inertial, and positioning sensors), we rely on a $\mathrm{KF}$ approach, in which the position and attitude of the UAVs are updated from the inertial and positioning sensors, allowing us to predict the coordinates of the runway at a future time in the image, using current runway coordinates. This requires us to track the attitude of the UAV (through its Euler angles: pitch, roll, and yaw), and compute the position of the runway on the image through a mapping that is a function of rotation, translation, and camera distortion. We can thus write the runway position estimation problem as a norm-constrained KF [26], with the following dynamical model:

$$
x_{k}=(a, b, c, d)^{T} \in \boldsymbol{R}^{4}
$$

$$
x_{k+1}=A x_{k}+B u_{k}+\zeta_{k}
$$

$$
y_{k+1}=C_{k} x_{k}+\eta_{k}
$$

$$
\begin{aligned}
& \zeta_{k} \sim \mathcal{N}(0, Q) \\
& \eta_{k} \sim \mathcal{N}(0, U)
\end{aligned}
$$

$$
\text { s.t. }\left\|x^{T} \tilde{A} x\right\|=1
$$

$$
a \geq 0
$$

where the state $x_{k}$ consists the parameters of the runway lines, $y_{k}$ is measurements of the projection of the lines in the imaging plane, and $\zeta_{k}, \eta_{k}$ are model and measurement noise. The noise in the model could result due to, for example, deviations from the infinite parallel line assumption, and the measurement noise could arise because the measurement is obtained through a segmentation process. The last two constraints enforce uniqueness of the our representation for the lines. The matrices above are defined as

$$
A=\left[\mathrm{id}_{4 \times 4}\right]
$$

$$
B=0
$$

$$
U=0.01 \times \mathrm{id}_{4 \times 4}
$$

$$
Q=0.01 \times \mathrm{id}_{4 \times 4}
$$

$$
\tilde{A}=\left[\begin{array}{ll}
I_{2 \times 2} & 0_{2 \times 2} \\
0_{2 \times 2} & 0_{2 \times 2}
\end{array}\right]
$$

In the above definitions, the matrix $I_{i \times i}$ denotes the identity matrix of dimension $i$. Therefore, our dynamic model is that the parameters of the line do not change (up to modeling noise). The initial state estimate $x_{0}$ is chosen to be the output of the runway detection procedure outlined in Sec. II.B, which can be projected to the frame of the world by estimates of the altitude and orientation of the UAV. The crucial element of this dynamic model is the observation matrix $C_{k}$, which maps the Earth frame coordinates to the camera coordinates. In the following section, we derive the observation matrix $C_{k}$ as function of the attitude, position and camera parameters, which give rise to the matrices $R_{k}, T_{k}$ and the projection $\Pi$.

\section{B. Geometric Camera Model, Parameters, and Measurement Matrix}

Cameras have an intrinsic geometric model [27] that describes the mapping between the Earth frame coordinates and the camera coordinate system. This mapping depends on the optical properties, orientation, and position of the camera, as illustrated in Fig. 6.

The transformation between the Earth frame coordinates and the camera coordinate system involves multiple elementary transformations: rotation, denoted by a $3 \times 3$ rotation matrix, and translation, denoted by a $3 \mathrm{D}$ offset vector $t$ with respect to a reference point in the Earth frame. More precisely, the mapping between any point of coordinates $p=(x, y, z)^{T}$ in the Earth frame and its counterpart 


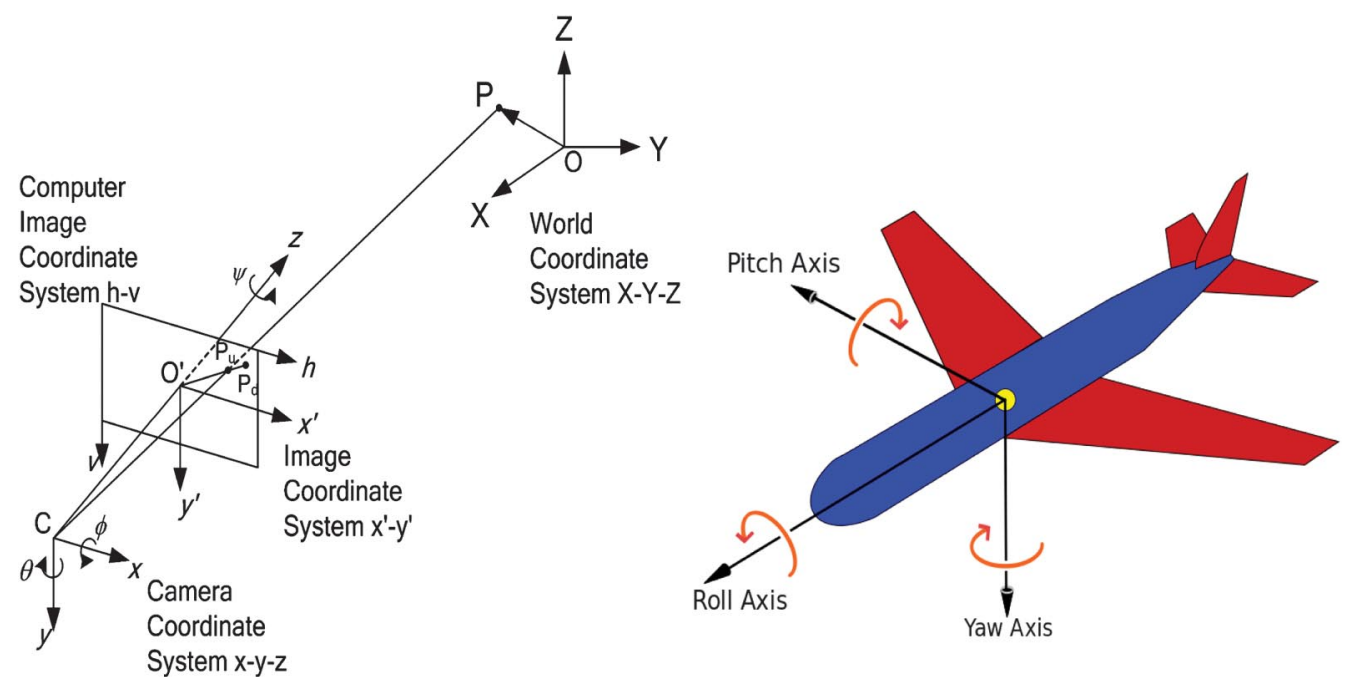

Fig. 6 Left: Projection from 3D point to 2D. Right: UAV coordinate system.

coordinates $p^{\prime}=\left(x^{\prime}, y^{\prime}\right)^{T}$ in the image is described by the following equation:

$$
\begin{gathered}
\left(\begin{array}{l}
x_{\mathrm{cam}} \\
y_{\mathrm{cam}} \\
z_{\mathrm{cam}}
\end{array}\right)=\kappa[R \mid t]\left(\begin{array}{l}
x \\
y \\
z \\
1
\end{array}\right)=\kappa\left[R\left(\begin{array}{l}
x \\
y \\
z
\end{array}\right)+\left(\begin{array}{l}
t_{x} \\
t_{y} \\
t_{z}
\end{array}\right)\right] \\
\left(\begin{array}{l}
x^{\prime} \\
y^{\prime}
\end{array}\right)=\Pi\left(\begin{array}{l}
x_{\mathrm{cam}} \\
y_{\mathrm{cam}} \\
z_{\mathrm{cam}}
\end{array}\right)=\left(\begin{array}{l}
x_{\mathrm{cam}} / z_{\mathrm{cam}} \\
y_{\mathrm{cam}} / z_{\mathrm{cam}}
\end{array}\right)
\end{gathered}
$$

In the above equation, the coordinates $\left(x^{\prime}, y^{\prime}\right)$ of a point on the image is obtained by a projection $\Pi$, which denotes the projection of points represented in the camera frame to the plane perpendicular to the direction of the camera with distance 1 away from the optical center of the camera. It assumes that $z_{\mathrm{cam}}$ is the distance from this imaging plane. By an argument of similar triangles, the projection is given by division by the last coordinate as seen above. The matrix $\kappa$ is a function of the intrinsic camera parameters: focal length, pixel size, and position of the principal point. Such parameters can be obtained by calibrating the camera [28], for example, using a calibration tool box [29]. The matrix $\kappa$ corresponds to the composition of three transformations: $\kappa=\kappa_{1} \times \kappa_{2} \times \kappa_{3}$, respectively corresponding to translation, scaling, and shear.

$$
\begin{aligned}
\kappa_{1} & =\left[\begin{array}{lll}
1 & 0 & x_{o} \\
0 & 1 & y_{o} \\
0 & 0 & 1
\end{array}\right], \quad \kappa_{2}=\left[\begin{array}{ccc}
f_{x} & 0 & 0 \\
0 & f_{y} & 0 \\
0 & 0 & 1
\end{array}\right], \\
\kappa_{3} & =\left[\begin{array}{ccc}
1 & s f_{x} & 0 \\
0 & 1 & 0 \\
0 & 0 & 1
\end{array}\right]
\end{aligned}
$$

Using (12) and find the equivalent transformation that could be applied to the vector $(a, b, c)$ instead of the point $p=(x, y, z)^{T}$ using Eq. (26).

Because the points that we track are on the ground, we can assume that their altitude is zero $Z=0$ (by setting the altitude reference to the runway altitude). Therefore, we can also write a linear relationship between the runway equation coefficients in the Earth frame and in the camera frame:

$$
\left(\begin{array}{l}
a^{\prime} \\
b^{\prime} \\
c^{\prime}
\end{array}\right)=E\left(\begin{array}{l}
a \\
b \\
c
\end{array}\right)
$$

given that $r_{i}$ and $k_{i}$ are the $i$ th column of $R^{t}$ and $\kappa$, respectively. Then

$$
E=\left(\begin{array}{lll}
e_{1} & e_{2} & e_{3}
\end{array}\right)^{-T}
$$

where

$$
e_{1}=\left(\begin{array}{l}
k_{1} \cdot r_{1} \\
k_{2} \cdot r_{1} \\
k_{3} \cdot r_{1}
\end{array}\right), \quad e_{2}=\left(\begin{array}{l}
k_{1} \cdot r_{2} \\
k_{2} \cdot r_{2} \\
k_{3} \cdot r_{2}
\end{array}\right), \quad e_{3}=\left(\begin{array}{l}
k_{1} \cdot t \\
k_{2} \cdot t \\
k_{3} \cdot t
\end{array}\right)
$$

It can be written as dot product between the columns of the internist parameters matrix and the extrinsic parameters matrix (i.e., rotation and translation).

Now, because we have two parallel lines that represent the runway in the world coordinate system as in 12, we get for "Line 1" and "Line 2", respectively,

$$
\left(\begin{array}{l}
a^{\prime} \\
b^{\prime} \\
c^{\prime}
\end{array}\right)=E\left(\begin{array}{l}
a \\
b \\
c
\end{array}\right), \quad\left(\begin{array}{l}
d^{\prime} \\
e^{\prime} \\
f^{\prime}
\end{array}\right)=E\left(\begin{array}{l}
a \\
b \\
d
\end{array}\right)
$$

By concatenating the two vectors at the left-hand side, we can find a total transformation:

$$
\left(\begin{array}{l}
a^{\prime} \\
b^{\prime} \\
c^{\prime} \\
d^{\prime} \\
e^{\prime} \\
f^{\prime}
\end{array}\right)=C_{k}\left(\begin{array}{l}
a \\
b \\
c \\
d
\end{array}\right)
$$


where $C_{k}$ is a $6 \times 4$ matrix given by

$$
C_{k}=\left(\begin{array}{cccc}
e_{1} & e_{2} & e_{3} & 0_{(3 \times 1)} \\
e_{1} & e_{2} & 0_{(3 \times 1)} & e_{3}
\end{array}\right)
$$

where $e_{i}$ is the $i$ th column of matrix $E$. The detailed derivation of $C_{k}$ is shown in the Appendixes.

To conclude, matrix $C_{k}$ will be used in KF to project the lines from $3 \mathrm{D}$ to $2 \mathrm{D}$, using the lines' parameters. Afterward, these lines would be refined using the segmentation based on the energy model. Because a neighborhood around the lines should be estimated efficiently, matrix $C_{k}$ arises to provide a robust solution for that. At each instance $k$, the lines parameters $c$ and $d$ particularly are shifted with a proper distance in $3 \mathrm{D}$, to generate the neighborhood as straight lines around the edges of the runway in 3D. Then, they are projected into the image, using $C_{k}$, resulting a trapezoidal region parameterized by $\ell(x)$ defined in Sec. II.A. $\ell(x)$ would increase from up to bottom around the runway edges, due to the perspective projection, as originally $\ell(x)$ is uniform in $3 \mathrm{D}$.

\section{Camera Model Construction}

The measurement matrix $C_{k}$ derived in the previous section relies on matrices $R$ and $t$, which are a function of the attitude and position of the UAV. We use a North, East, and Up orientation convention for the Earth frame, which requires some sign changes for angles and translation coefficients, in order to transform coordinates and rotations expressed in a right-handed coordinate system into a left-handed coordinate system. The orientation of the camera with respect to the Earth frame is the composition of a rotation of $-\pi / 2$ with respect to the $x$ axis of the UAV, with the rotation transforming the UAV coordinates to the Earth frame coordinates $R=R y(-\theta) R x(\phi) R z(\Psi)$, where $\Psi, \phi, \theta$ represent the Euler angles (respectively, roll, pitch, and yaw angles). Each elementary rotation is associated with a rotation matrix:

$$
\begin{aligned}
R_{z}(\Psi)= & {\left[\begin{array}{ccc}
\cos (\Psi) & -\sin (\Psi) & 0 \\
\sin (\Psi) & \cos (\Psi) & 0 \\
0 & 0 & 1
\end{array}\right], } \\
R_{y}(-\theta)= & {\left[\begin{array}{ccc}
\cos (\theta) & 0 & -\sin (\theta) \\
0 & 1 & 0 \\
\sin (\theta) & 0 & \cos (\theta)
\end{array}\right], } \\
R_{x}(\phi)= & {\left[\begin{array}{ccc}
1 & 0 & 0 \\
0 & \cos (\phi) & -\sin (\phi) \\
0 & \sin (\phi) & \cos (\phi)
\end{array}\right] }
\end{aligned}
$$

The translation vector mapping the origin of the Earth frame coordinates to the origin of the UAV coordinates requires the following measurement data:

1) The altitude of the UAV, denoted by $a_{t}$, and the altitude of the origin of the Earth frame, $a_{0}$

2) The latitude and longitude of the UAV, denoted by $\theta_{\operatorname{lat}_{t}}, \theta_{\operatorname{lng}_{t}}$

3 ) The latitude and longitude of the origin of the Earth frame, denoted by $\theta_{\text {lat }_{0}}, \theta_{\operatorname{lng}_{0}}$

4) The radius of the Earth $\rho$

At time $t$, the translation vector mapping the Earth frame and the UAV frame origins is given by

$$
t=\left[\begin{array}{c}
\rho \cdot\left(\theta_{\operatorname{lng}_{t}}-\theta_{\operatorname{lng}_{0}}\right) \cdot \cos \left(\frac{\theta_{\text {lat }_{t}}+\theta_{\text {lat }_{0}}}{2}\right) \\
a_{0}-a_{t} \\
\rho \cdot\left(\theta_{\mathrm{lat}_{t}}-\theta_{\mathrm{lat}_{0}}\right)
\end{array}\right]
$$

Because the Euler angles estimated by the IMU are not very accurate, we rely on the images captured by the camera to estimate the translation and rotation changes more finely between consecutive frames. This approach is detailed in Sec. III.D. In an actual implementation of the system, we can rely on a fusion of inertialcamera-based measurements to estimate the rotation and translation changes.

Now, we mention how the state of the EKF initialized. Basically, in $2 \mathrm{D}$ (i.e., the image is used), the detection algorithm gives the location of the runway throughout the points $p 1, \ldots, p 4$, and then the lines parameters are retrieved using Eqs. (12) and (13). Then by using the reading of the GPS, altitude, and rotation of the UAV, for the detection moment, Eq. (33) is used to find the lines parameters in 3D, which initializes the filtering process.

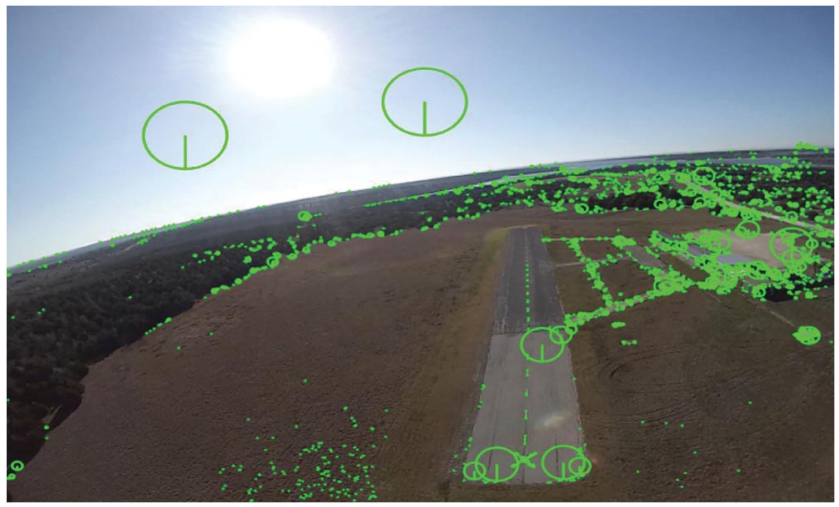

Fig. 8 Feature points detected by Harris detector.
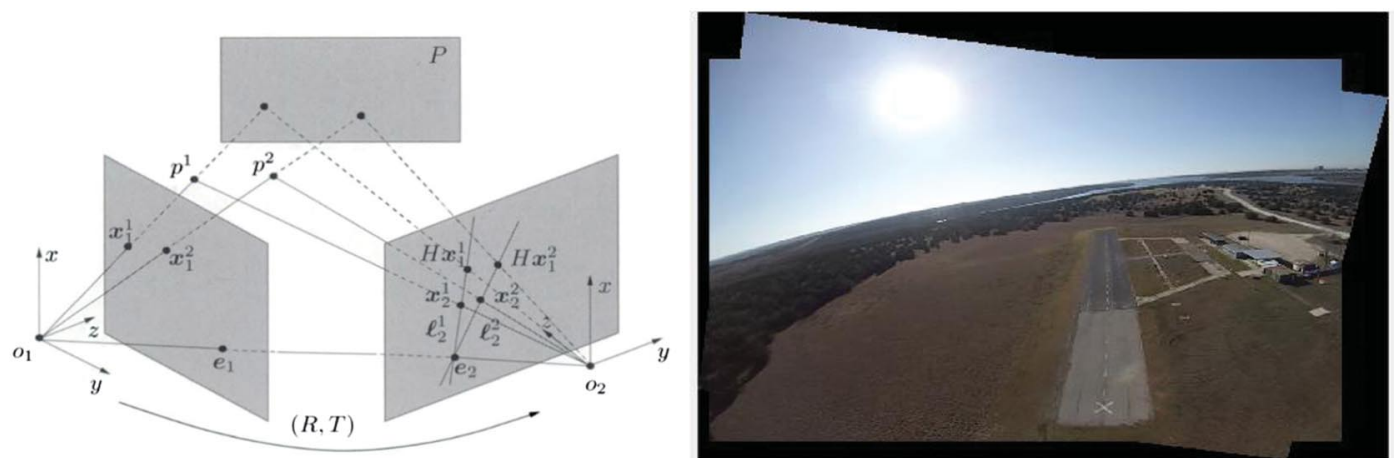

Fig. 7 Planar homography can be estimated using matched points between two frames, and then used to stitch the images. 


\section{Algorithm 2: $\quad$ Relative motion recovery}

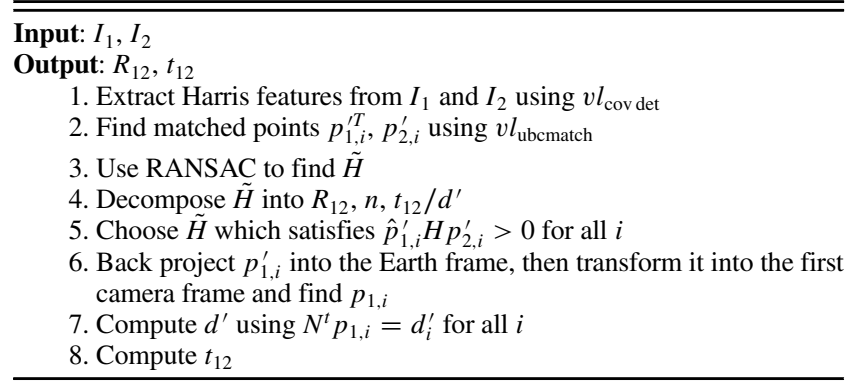

\section{Motion Recovery from Images}

The camera is a visual sensor that can be fused in implementation to estimate the motion at a certain time. In other words, it can substitute using the IMU and GPS, where it surpass any errors due to turbulence in the flight or bad synchronization with video. Accordingly, an imagebased algorithm is used to estimate translation and rotation changes between frames, and then composing such resultant transformation to find the final motion estimate at a certain time of the flight with respect to the Earth frame.

The image-based algorithm follows an assumption, according to camera pinhole model, that images of the same scene can be stitched together if there exists one planar homography $H$ that maps adjacent images [27] (refer to Fig. 7). The left subfigure shows that a planer homography maps the left image to the right image, whereas the right subfigure shows the two frames stitched together using the estimating planar homography. This assumption holds as long as the scene in the Earth frames appears to be flat in the image plane, which is achieved due to capturing images while flying at high altitude. A set of points in $I_{1}$, called feature points (refer to Fig. 8 ) should match with some points in the next image $I_{2}$, to find $H$. Feature points are detected such that they are discriminant and can be matched across frames, where in such aerial images, the corners of the runway on the ground, for instance, can be used more efficiently in the matching more than the points in the sky (refer to Fig. 8). Among different feature detectors, we found that Harris detector can provide features of the borders of the runway, which can make the matching process more efficient and accurate. Let us denote the features or set of interest points in $I_{1}$ and $I_{2}$ by $p_{1, i}^{\prime}, p_{2, i}^{\prime}$, respectively, where $i$ indexes the interest points. Choosing a different set of matched points to compute homography leads to different results, especially with the existence of outliers. Accordingly, RANSAC is used in this process to avoid such errors. The following system can be solved to estimate the homography,

$$
\hat{p}_{1, i}^{\prime} H p_{2, i}^{\prime}=0 \text {, for all } i
$$

where the hat operator denotes to cross product. Because the former formula applies for identity calibration matrix, the resultant $\mathrm{H}$ should be premultiplied by $\kappa^{-1}$ and multiplied by $\kappa$, and is denoted by $\tilde{H}$. Afterward, it can easily be verified that $\tilde{H}$ transforms the first frame to the second one. Refer to Fig. 7. Finally, $\tilde{H}$ can be decomposed as follows:

$$
\tilde{H}=R_{12}+\frac{t_{12} n^{T}}{d^{\prime}}
$$

where $R_{12}$ and $t_{12}$ are the rotation and translation between two adjacent frames, respectively, $n=\left[n_{1}, n_{2}, n_{3}\right]^{T}$ is the unit normal vector of the $3 \mathrm{D}$ plane in the Earth frame with respect to the first camera frame, which is related to the first image, and $d^{\prime}$ denotes the distance from the 3D plane to the optical center of the camera.

Decomposition gives four solutions, and we choose the one that satisfies $\hat{p}_{1, i}^{\prime} H p_{2, i}^{\prime}>0$ for all $i$. Knowing that $t_{12} / d^{\prime}$ is retrieved from the decomposition, one can compute $d$ using $N^{T} p_{1, i}=d_{i}$, and we take an average of the $d_{i}$ to compute the depth $d . p_{1}$ is the 3D corresponding to $p_{1}^{\prime}$ in the first camera frame, related by $p_{i}=\lambda_{i} p_{i}^{\prime}$, and $\lambda_{i}$ states the ambiguity inhibited when projecting points from 3D points in $2 \mathrm{D}$, according to Eq. (C1).

Back projection is applied to $p_{1}^{\prime}$ to find its corresponding in the Earth frame, using Eqs. (C6) and (C7), where the assumption is zero for the third component where it lies on the runway. After that, a transformation is applied to the point in the Earth frame, using the rotation $R_{1}^{T}$ and translation $-R_{1} t_{1}$ associated with image frame containing $p_{1}^{\prime}$, to find $p_{1}$, where the last component of the translation vector is set to be the altitude measured by the altimeter, and then $d^{\prime}$ is computed. For more details, the reader may refer to [27]. The algorithm for relative motion estimation between adjacent frames is summarized in Algorithm 2.

In Algorithm 2, Step $\overline{2}$, the interest points and features are computed using VLFeat [30]. We use the Harris interest point detector and the SIFT features to describe the regions of the image local to the interest points. We use the matching procedure proposed by Lowe [31] to match SIFT features; that is, for each feature in $I_{1}$ the distance between it and each feature in $I_{2}$ is computed. The best match (with smallest distance) is selected as a match if the ratio of the lowest distance to the second smallest distance is greater than a given threshold, so as to reject ambiguous matches. Note that for speed, one can use SURF [32] for the feature and FAST corner detector [33] to achieve speed ups of the traditional SIFT and Harris detectors, respectively, for real-time speeds.

For standard motion composition, we would use the rotation $R_{12}$ and translation $t_{12}$ between two adjacent frames, given the motion $\left(R_{1}, t_{1}\right)$ induced with the first frame, to find the final motion $\left(R_{2}, t_{2}\right)$ at the second frame. This process is mathematically expressed as follows:

$$
t_{2}=R_{1} t_{12}+t_{1}, \quad R_{2}=R_{1} R_{12}
$$

This procedure can be generalized to find the rotation and translation at any frame, given that $R_{0}=\mathrm{id}_{3 \times 3}$ and $t_{0}=0$ at the initial location in the Earth frame. Hence, the measurement matrix can be computed and used in our dynamical model throughout KF.

\section{Experimental Results}

\section{A. Experimental Validation}

We now evaluate the performance of the algorithm described in this paper over different video samples generated by UAV filmingsimulated approaches to the Austin Radio Control Associates (ARCA) runway in Austin, TX. These samples are obtained using an electric-powered Bormatec Maja fixed-wing UAV, from the Distributed Sensing Systems Laboratory of the University of Texas at Austin.

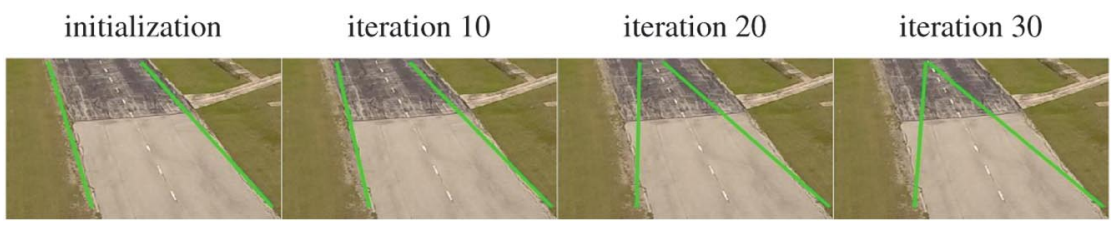

Fig. 9 GD evolution given near edges initializations using the nonspatially variant mean calculation (2). This figure illustrates the convergence of the gradient-based minimization of Eq. (3). 


\section{B. Segmentation Results}

The gradient descent evolution is illustrated in Fig. 9 using one image over a number of iterations. This figure shows that minimizing the energy function using nonspatially variant mean (2) is inferior to minimization based on spatially variant mean (1), which more robustly tracks the runway because the latter is more robust to

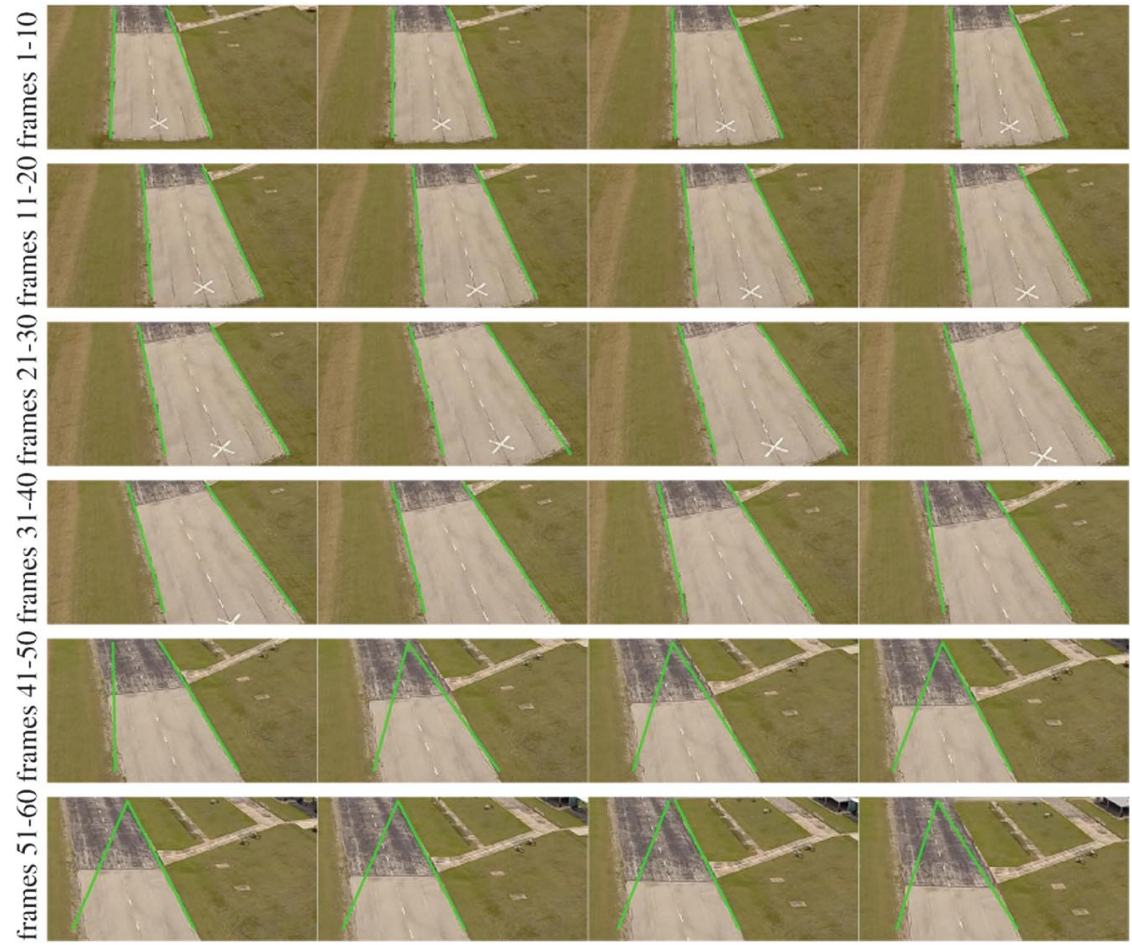

Fig. 10 Output of segmentation algorithm over a sequence of images based on the nonspatially variant mean calculation.

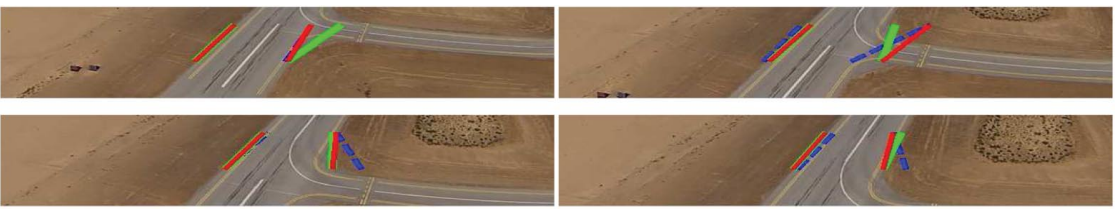

Fig. 11 Visualization of the KF performance in tracking the runway edges.
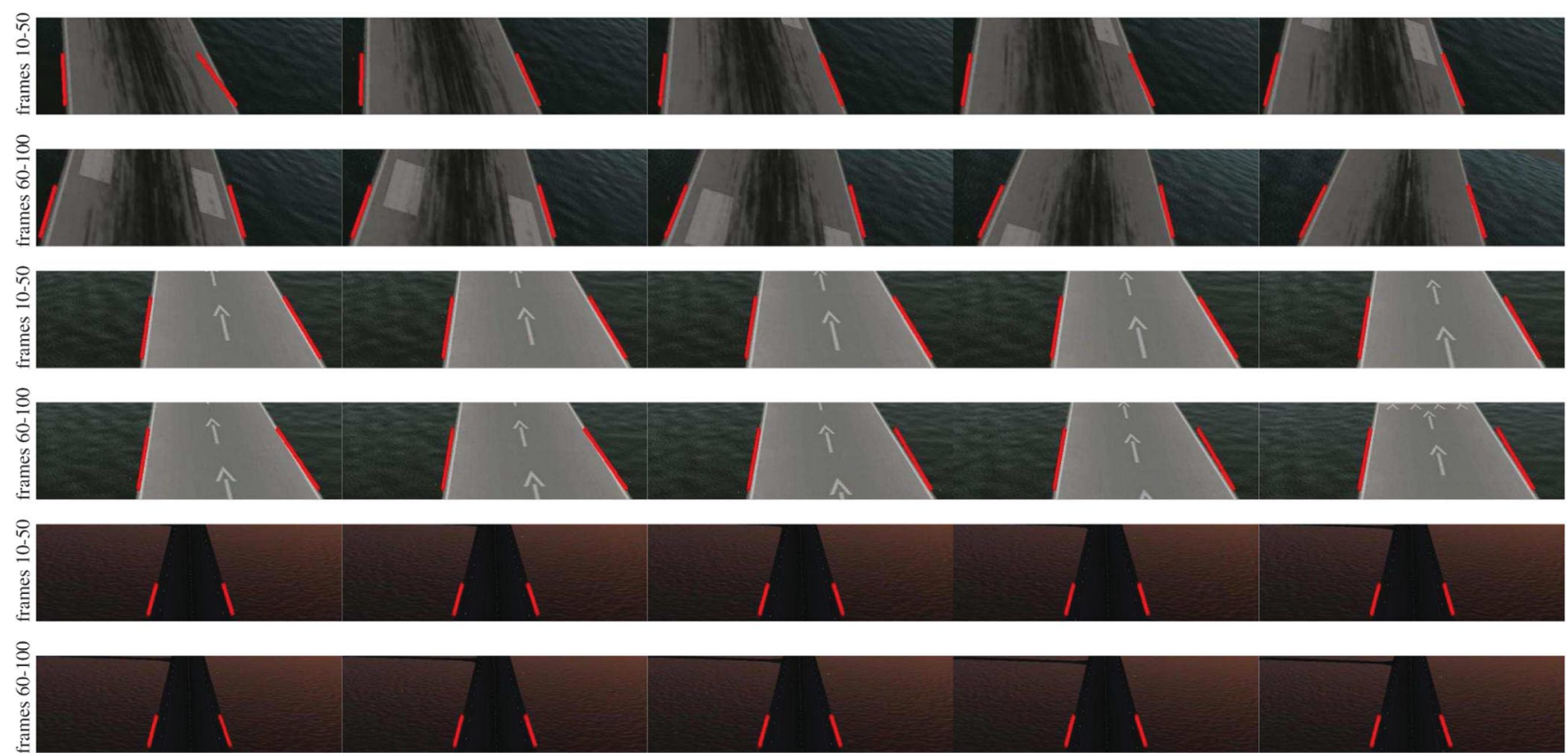

Fig. 12 Video sequences from the Xplane flight simulator under different illuminations. This figure shows three different lighting and environmental conditions, each represented over two rows. 
nonuniformities in the axis of the runway, such as ground markings or tire tracks.

In Fig. 10, we only apply the runway segmentation algorithm over a sequence of images (without tracking) whereby initialization at any frame would be the result of the segmentation algorithm from the previous frame. This sequence shows that energy based on nonspatially variant mean calculation (2) fails in segmenting the runway whenever the variations in image statistics along the runway axis are variant.

\section{Tracking Results}

Visualization of the KF performance in tracking the runway edges is shown in Fig. 11. We consider four consecutive time steps (left to right and up to down): blue line, predicted runway edge locations (from previous step); green line, runway edge locations (measurements) using vision-based segmentation (outlined in Sec. III of the present paper); red lines, estimation (output of the KF).

Figure 12 shows the performance of the tracker in [25], while Figs. 13 and 14 show how the runway is tracked under different lighting conditions, demonstrating the robustness of the proposed approach. The red lines show the output of the KF (which fuses the dynamics of the UAV to the observations of the runway detection algorithm) over a sequence of images. Reader may refer to [34] for videos.

Figure 15 shows a comparison of the performance of the two energy functions investigated in this paper. The motion information of the UAV helps the algorithm converge, though the constant velocity model (without Kalman filtering) performs adequately in low-turbulence conditions. The red lines show the estimated location of the runway using $\mathrm{KF}$ where an infinite weight on the prediction, whereas the measurements are in green and it is not considered in the filtering process although it is perfect. The 3D-based dynamical model outperforms the 2D-based model.

Setting infinite weight on the prediction in both models and having a perfect measurement, which its uncertainty, is extremely high so that only the prediction is affecting performance of the KF. In this experiment, although the measurement is perfect, its noise is set to be high only to show the robustness of the prediction model of tracking in $3 \mathrm{D}$.

\section{Quantitative Evaluation}

In Table 1, a comparison is conducted between the ground truth runway position and the performance of the nonspatially variant mean-based model (2), the spatially variant mean-based model (1), and a HT with Canny edge detector approach. The performance metric used is the root mean square error in pixel position, using the ground truth as a reference, averaged over sequences of 50 consecutive frames.

The computational time results differ according to the hardware implementation. For the segmentation process, it boils down to a line integral over line segment, in addition to Kalman filtering that involves only matrix multiplications. This complete process requires $30 \mathrm{~ms}$ using an Odroid U3X, which is considered as a small computer. Hence, the algorithm is fast enough to run at $20 \mathrm{~Hz}$ (or slightly more), which is comparable to the frequencies used by the high- and low-level control loops of actual consumer-grade UAVs $(10 \mathrm{~Hz}$ for Pixhawk-based systems). For future work, a computer stick and NUC intel would be used to further improve the computational speed.

\section{Discussion}

The experimental comparison against HT and Canny edge detection can raise a question of a fairer comparison that would be against graph cut [35] or other appearance-based segmentation algorithms, which can also be quite fast. Basically, looking at such based-region segmentation algorithm was the first step to approach the runway detection. However, it requires user supervision by selecting a number of seed points to start clustering all pixels in the image (expensive) into groups based on their appearance similarities.
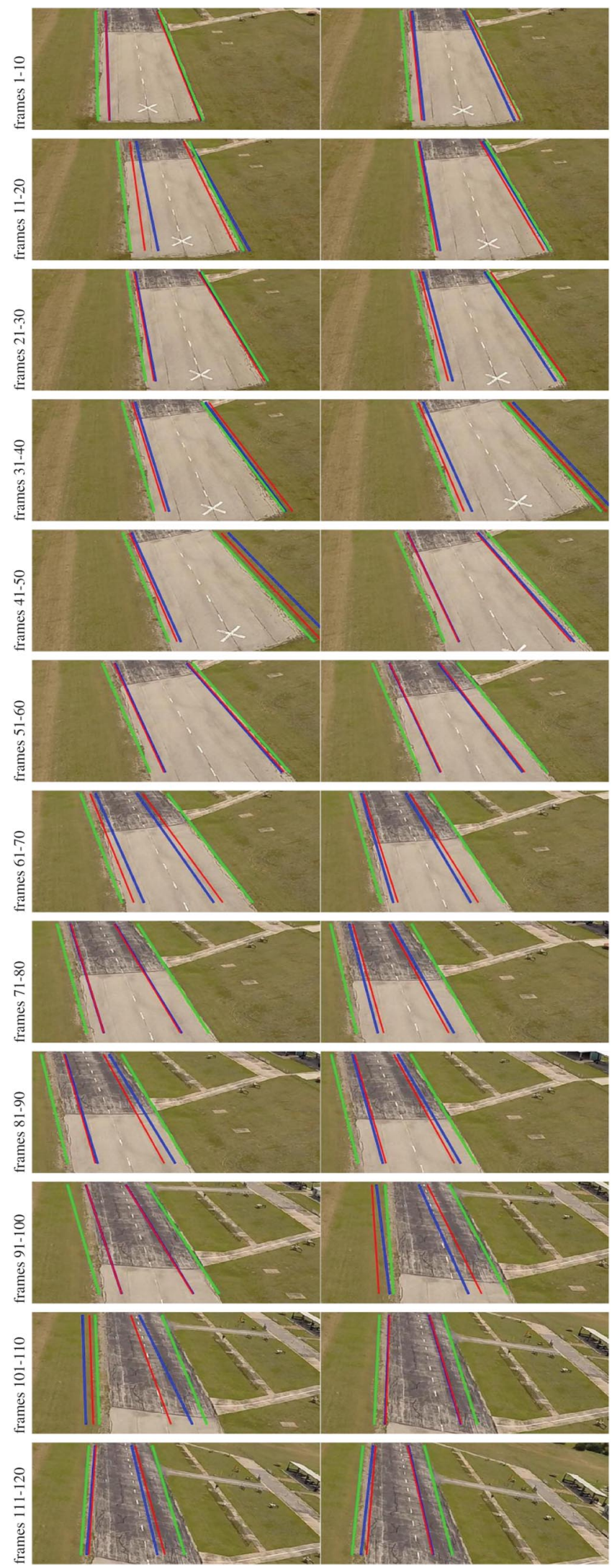

Fig. 13 Video sequence 1 showing the tracking output using 3D-based tracking model with spatially variant mean energy model for segmentation.

In more detail, this can be involved in the detection and segmentation steps. First, for the detection step. Our approach is generic enough to find edges directly without any other postprocessing step, such as edge detectors. Other methods, such as graph cut, would similarly use color-based segmentation energy function; with excellent 

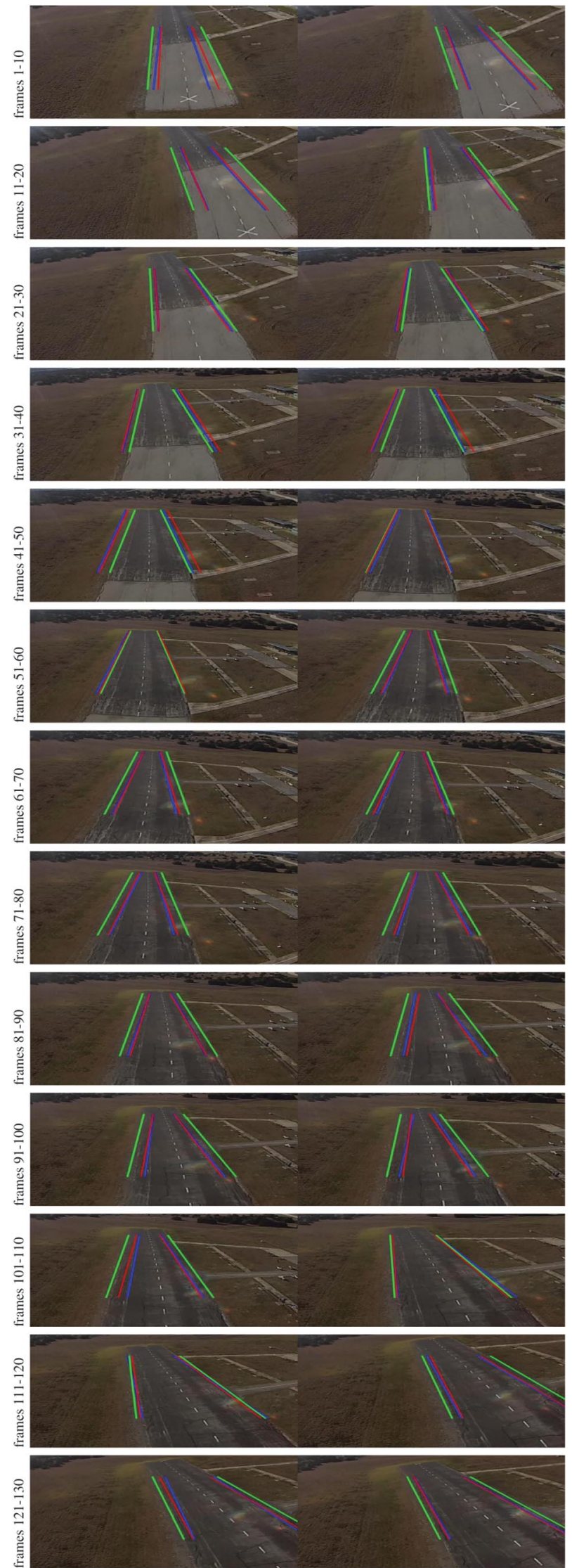

Fig. 14 Video sequence 2 showing the tracking output using 3D-based tracking model with spatially variant mean energy model for segmentation.

conditions of not having any clutters, it would be subjected to loss of detection of the runway edges under difficult illumination conditions, whereas our method finds candidates of edges that could form the
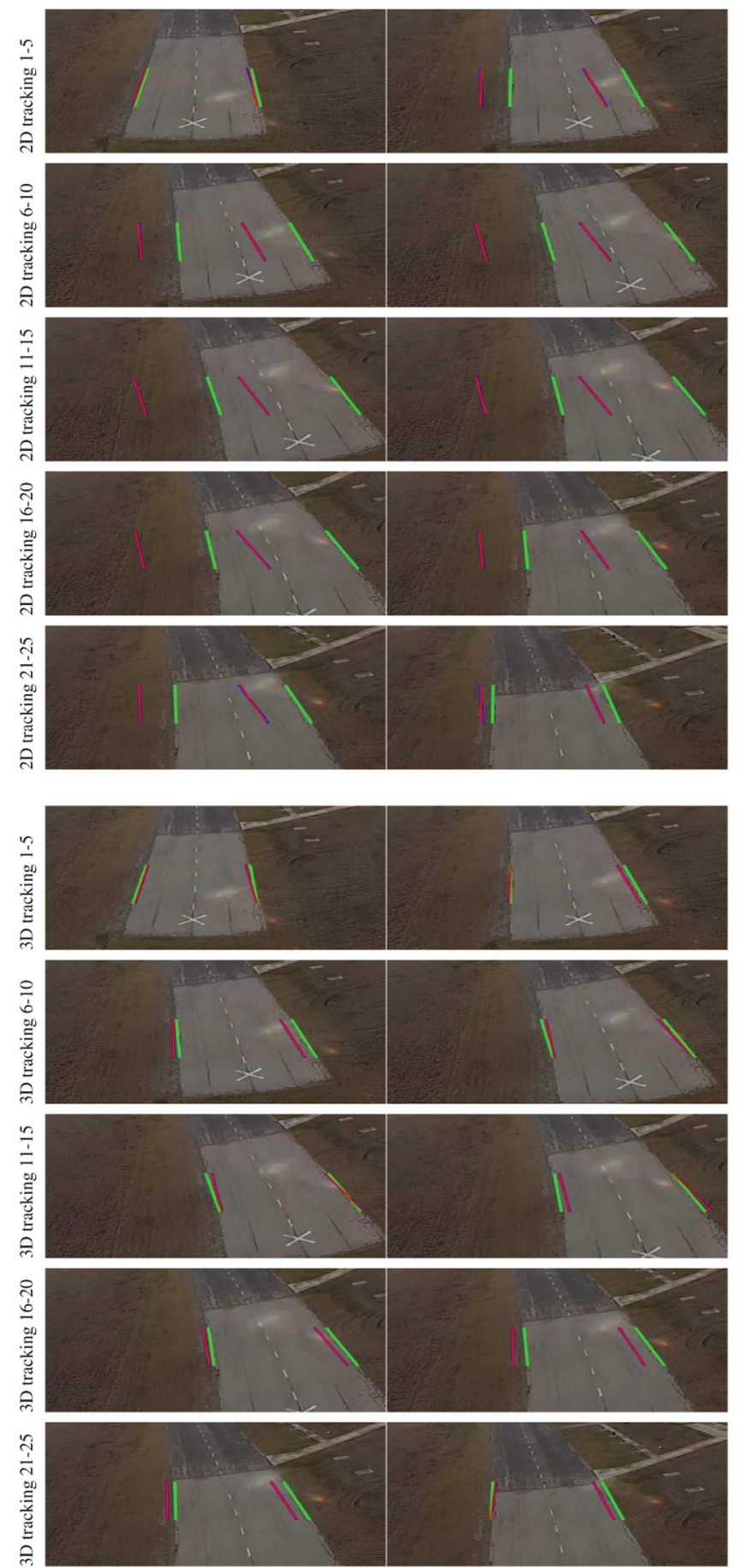

Fig. 15 Comparison between the performance of the spatially (related to 3D tracking) and nonspatially (related to 2D tracking) variant meanbased models.

runway and then find their correlation response. If we assume that graph cuts are free of miss detections, then an extra step is required to find the edges of the region of interest of the runway (given that we restricted the search region for the graph cut method), and this would give many possible candidates, including the white land marks on the

Table 1 Average pixel error in runway position estimation

\begin{tabular}{lccc}
\hline \hline Sequence & $\begin{array}{c}\text { Spatially } \\
\text { variant mean }\end{array}$ & $\begin{array}{c}\text { Nonspatially } \\
\text { variant mean }\end{array}$ & $\begin{array}{c}\text { Hough transform and } \\
\text { Canny edge detector }\end{array}$ \\
\hline 1 & $6.9 \pm 3.6$ & $30.9 \pm 24.5$ & $54.3 \pm 99.4$ \\
2 & $7.3 \pm 2.9$ & $32.9 \pm 22.6$ & $57.9 \pm 39.9$ \\
\hline \hline
\end{tabular}


runway thresholds. To conclude, our detection method is robust to different illumination conditions, weather conditions, and runway marking layouts.

Second, for the segmentation. Graph cuts method requires from the users initial seeds, which are difficult to set. Hence, our method outperform in terms of user-free operation. Also, assuming that we are interested in two labeled segmentations, graph cuts based-color based segmentation methods, such as on k-means, histograms, or region competition, can end up at a local minima (causing a false detection). This can occur in the presence of landmarks, road marks, taxiways, clutter, or because of changes in illumination. Also, such results would violate the geometric constraint of having a trapezoidal region of the runway. On the other side, our algorithm is generic and can overcome the aforementioned nuisances and reduces the space of parameters to only four points, preserving the geometric properties of a trapezoidal shape.

The following figures show the challenge when using the graph cut method. On the left of Fig. 16a are a four regions output from graph cut, where the runway is clustered with the sun, part of the horizon, and part of the ground clutters. With no prior information, the runway cannot be extracted. On the right, the contour output of the clustered image, based on built-in tuned threshold, the runway cannot be located. Even when choosing to segment three regions, the runway is missed.

This illustrates a significant problem in making use of the graph cut algorithm in designing a fully automatic detection and segmentation algorithm; namely, it is difficult to choose a priori the number of regions (or seeds) to choose. Choosing a small number of regions leads to an undersegmentation, and a large number leads to an oversegmentation of the runway. In general, the number will vary with the scenery present, making it nearly impossible to choose the "correct number" a priori. Moreover, there is no guarantee that the runway will appear as a segment for any number of regions chosen. Even if it does, one would still need to ascertain which of the segments is the runway, requiring further postprocessing. These problems are the motivation for our model-based detection and segmentation scheme that essentially fits a simple geometric model of the runway to the image(s). As Figs. $16 \mathrm{~b}$ and $\underline{17 \mathrm{~b}}$ show, our method obtains the correct result automatically.

In the upper row of Fig. 17a, the region of interest is specified for graph cut by the user. Two regions output from graph cut, and still the runway is not detected. In the lower row of Fig. 17a, there is same input as before, but with different output, due to the randomized initialization inherited in the graph cut algorithm.
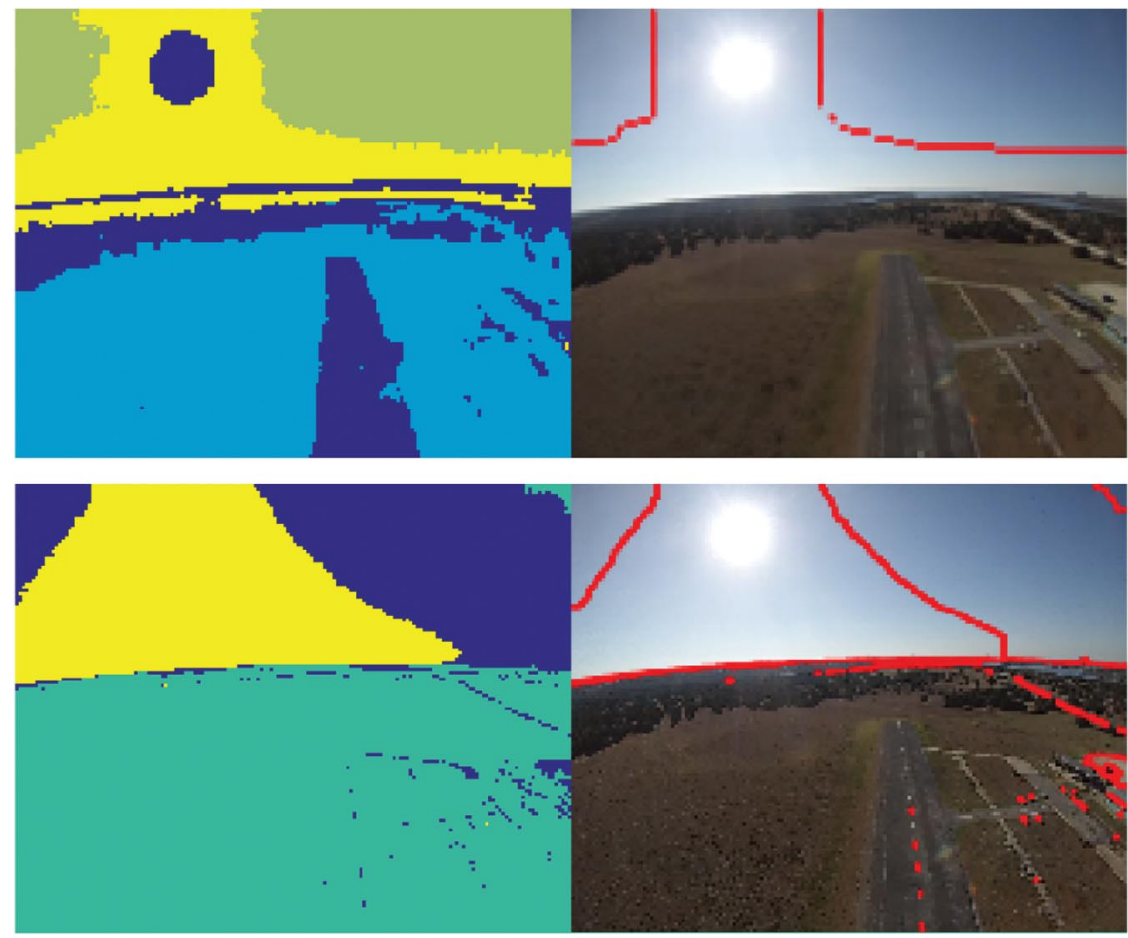

a) Graph cuts' output

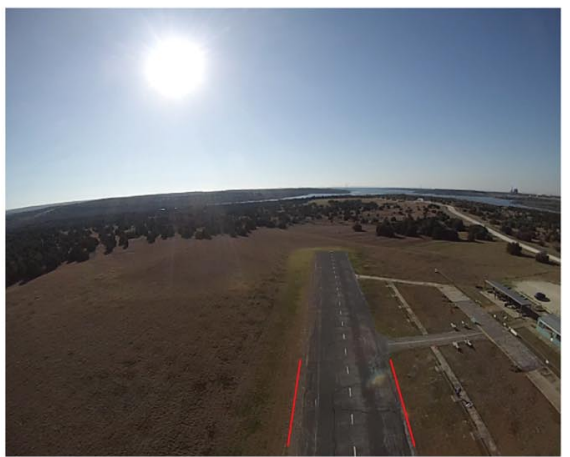

b) Our detection algorithm output

Fig. 16 Comparison of our segmentation and detection method with graph cuts. a) Result of graph cuts for four regions (top) and three regions (bottom). Graph cuts are highly sensitive to the number of regions input by the user. Thus, in some cases the runway is obtained and sometimes it is not. The images on the left are the segmentation and the images on the right are the corresponding boundaries. b) Our method correctly detects and segments the runway automatically - the main motivation for our approach. 

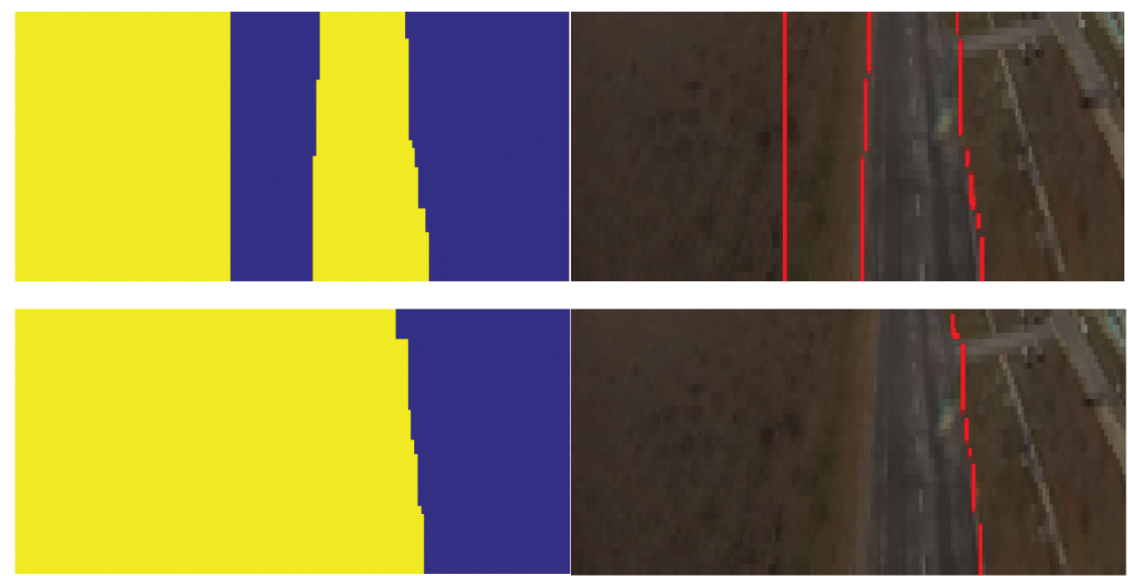

a) Graph cuts' output for a specified region of interest

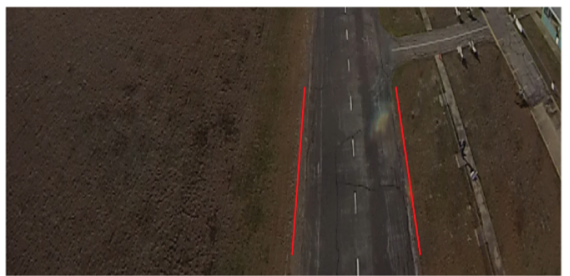

b) Our detection algorithm output

Fig. 17 Comparison of our method with graph cuts. a) Even in the case that the image is a simple bimodal image where the correct number of regions is 2 , and the graph cut algorithm is given the correct number, the results depend on the location of the seeds. The top and bottom show the results with two different initial seeds. b) Our detection and segmentation algorithms are able to achieve the correct result, because the detection process is a robust global search, which gives good initialization to the gradient descent for the segmentation scheme, which is also robust to local minima because the optimization is over a space that is very low dimensional.

\section{Conclusions}

This paper introduces a robust runway detection and tracking algorithm that can be used for real-time UAV control in landing conditions. Unlike recent approaches to runway detection using computer vision, our approach requires no rendered computer model of the shape and appearance of the runway, making it very robust to different types of runways and lighting conditions. Generating rendered computer models under all possible illumination/weather conditions, runway geometries, and runway materials (grass, concrete, asphalt, dirt) is not scalable, especially because small UAVs can operate from a large number of small airstrips or private roads. Our approach uses a simple model of the local geometry of the runway and makes no assumption on the appearance of the runway other than the dissimilarity of the runway with respect to its surrounding and the assumption that the runway is straight. We show that a combination of runway position estimation (through the minimization of an energy function derived from the runway model) and Kalman filtering using inertial or visual attitude information allows us to efficiently and robustly track the runway in real time. The performance of the runway detection algorithm is sufficiently good to use it directly (without any inertial measurements) for most runway landing applications.

\section{Appendix A: Computation of the Gradient of $E$}

In this section, we show the details for the computation of the gradient of $E$ with respect to $\boldsymbol{d}=\left(d_{1}, d_{2}, d_{3}, d_{4}\right)$. Using the chain rule, we see that

$$
\nabla E(\boldsymbol{d})=-(u(\boldsymbol{d})-v(\boldsymbol{d}))(\nabla u(\boldsymbol{d})-\nabla v(\boldsymbol{d}))
$$

where $\nabla$ denotes the gradient with respect to $\boldsymbol{d}$.

It remains to compute the gradient of $u$ and $v$ with respect to $\boldsymbol{d}$, which are defined by

$$
u(\boldsymbol{d})=\frac{\int_{R} I(x) \mathrm{d} A}{\int_{R} \mathrm{~d} A}, \quad v(\boldsymbol{d})=\frac{\int_{R^{c}} I(x) \mathrm{d} A}{\int_{R^{c}} \mathrm{~d} A}
$$

where $R$ is the banded region inside the trapezoid determined by $d, R^{c}$ is the banded region outside the trapezoid determined by $\boldsymbol{d}$, and $d A$ is the area differential element. To compute the gradient, we may use the result [36], which states that the directional derivative of a functional

$$
e(R)=\int_{R} f(x) \mathrm{d} A
$$

is given by

$$
d e(R) \cdot h=\int_{\partial R} f(x) \mathrm{d} s
$$

where $h$ is a perturbation of the boundary of $R, \partial R$ is the boundary of $R$ that is allowed to vary, $d s$ is the arclength differential element, and $d e(R) \cdot h$ is the change in $e$ by perturbing $R$ by a perturbation $h$ (defined on the boundary of $\partial R$ ).

By applying the quotient rule and the previous result, one can show that

$$
\begin{aligned}
& \frac{\partial u}{\partial d_{j}}=\frac{1}{|R|} \int_{C_{L_{i}}}(I-u) h_{j} \cdot N \mathrm{~d} s \\
& \frac{\partial v}{\partial d_{j}}=-\frac{1}{\left|R^{c}\right|} \int_{C_{L_{i}}}(I-v) h_{j} \cdot N \mathrm{~d} s
\end{aligned}
$$

where $C_{L}:[0,1] \rightarrow R^{2}$ is the line segment corresponding to either side (right or left) of the trapezoid, $N$ is the unit outward normal to $C_{L}$, and $h_{j}$ corresponds to the perturbation of $C_{L_{j}}$ when the $j$ th vertex is perturbed.

$i=1,2$ and $i=3,4$ correspond to line segments at the left side and the right side, respectively. While $j=1,2,3,4$ correspond to the four variables that should be updated. Note that 


$$
C_{L_{i}}(t)= \begin{cases}\left(\begin{array}{c}
d_{1} \\
0
\end{array}\right)+\left(\begin{array}{c}
d_{2}-d_{1} \\
L_{2}
\end{array}\right) t & \text { if } i=1,2 \\
\left(\begin{array}{c}
L_{1}-d_{3} \\
0
\end{array}\right)+\left(\begin{array}{c}
d_{3}-d_{4} \\
L_{2}
\end{array}\right) t & \text { if } i=3,4\end{cases}
$$

and

$$
h_{j}=\frac{\partial C(t)}{\partial d_{j}}= \begin{cases}\left(\begin{array}{c}
1-t \\
0
\end{array}\right) & \text { if } j=1,3 \\
\left(\begin{array}{c}
t \\
0
\end{array}\right) & \text { if } j=1,4\end{cases}
$$

Because $N$ is the outward normal to $C_{L_{i}}$, it can be related to the tangent $T$ to $C_{L_{i}}$ by $N=J T$, where $J$

$$
J=\left[\begin{array}{cc}
0 & -1 \\
1 & 0
\end{array}\right]
$$

is the rotation matrix with $\theta=-90^{\circ}$ counterclockwise. Note also that

$$
T(t)=\frac{C_{L_{j}}^{\prime}(t)}{\left|C_{L_{j}}^{\prime}(t)\right|}
$$

where $\left|C_{L_{j}}^{\prime}(t)\right|$ is the speed of the point, $s$ denotes the arc length parameter,

$$
d s=\left|C_{L_{j}}^{\prime}(t)\right| d t
$$

and $L_{j}$ will denote the length of $C_{L_{j}}$. Combining Eqs. (A4), ( $\underline{\mathrm{A} 5}$ ), (A7), and (A8) results in

$$
N(t) \cdot h(t) d s=L_{2}(t-1) d t
$$

Then, the gradient direction at vertex $j=3$, for example, can be derived as follows:

$$
\begin{aligned}
\frac{\partial E}{\partial d_{3}} & =-L_{2}(u-v)\left[\frac{1}{|R|} \int_{0}^{1}(t-1)\left(I\left(C_{L_{2}}(t)\right)-u\right) \mathrm{d} t\right. \\
& \left.+\frac{1}{\left|R^{c}\right|} \int_{0}^{1}(t-1) I\left(\left(C_{L_{2}}(t)\right)-v\right) \mathrm{d} t\right]
\end{aligned}
$$

The same argument applies to the other $d_{i}$, and thus

$$
\begin{gathered}
\frac{\partial E}{\partial d_{1}}=L_{2}(v-u)\left[\frac{1}{|R|} \int_{0}^{1}(t-1)\left(I\left(C_{L_{1}}(t)\right)-u\right) \mathrm{d} t\right. \\
\left.+\frac{1}{\left|R^{c}\right|} \int_{0}^{1}(t-1)\left(I\left(C_{L_{1}}(t)\right)-v\right) \mathrm{d} t\right] \\
\frac{\partial E}{\partial d_{2}}=L_{2}(v-u)\left[\frac{1}{|R|} \int_{0}^{1} t\left(I\left(C_{L_{1}}(t)\right)-u\right) \mathrm{d} t\right. \\
\left.+\frac{1}{\left|R^{c}\right|} \int_{0}^{1} t\left(I\left(C_{L_{1}}(t)\right)-v\right) \mathrm{d} t\right] \\
\frac{\partial E}{\partial d_{3}}=L_{2}(v-u)\left[\frac{1}{|R|} \int_{0}^{1}(t-1)\left(I\left(C_{L_{2}}(t)\right)-u\right) \mathrm{d} t\right. \\
\left.+\frac{1}{\left|R^{c}\right|} \int_{0}^{1}(t-1) I\left(\left(C_{L_{2}}(t)\right)-v\right) \mathrm{d} t\right]
\end{gathered}
$$

$$
\begin{aligned}
\frac{\partial E}{\partial d_{4}} & =L_{2}(v-u)\left[\frac{1}{|R|} \int_{0}^{1} t\left(I\left(C_{L_{2}}(t)\right)-u\right) \mathrm{d} t\right. \\
& \left.+\frac{1}{\left|R^{c}\right|} \int_{0}^{1} t\left(I\left(C_{L_{2}}(t)\right)-v\right) \mathrm{d} t\right]
\end{aligned}
$$

This derivation applies for the special case where $u$ and $v$ are nonspatially variant. Generalizing the previous expressions for spatially variant means would require to calculate the mean in a neighborhood, at each point on the line segment, represented by a line that lies in the normal direction. Hence,

$$
\begin{aligned}
& u_{L_{i}}(t)=\frac{1}{\ell(t)} \int_{0}^{\ell(t)} I\left(C_{L_{i}}(t)-s \cdot N\right) \mathrm{d} s, \\
& v_{L_{i}}(t)=\frac{1}{\ell(t)} \int_{0}^{\ell(t)} I\left(C_{L_{i}}(t)+s \cdot N\right) \mathrm{d} s
\end{aligned}
$$

where $d s$ is the arclength differential element of the segment defined on $\left[C_{L_{i}}(t), C_{L_{i}}(t)-\ell(t) N\right],\left[C_{L_{i}}(t), C_{L_{i}}(t)+\ell(t) N\right]$ for $u_{L_{i}}(t), v_{L_{i}}(t)$, respectively.

\section{Appendix B: Computation of the Measurement Matrix $\boldsymbol{C}_{\boldsymbol{k}}$}

We derive the mapping between the parameters of a line in the ground plane of the world coordinate system and the parameters of the same line in the image plane.

A line given in the imaging plane, a 2D coordinate system, can be written as

$$
a^{\prime} x^{\prime}+b^{\prime} y^{\prime}+c^{\prime}=0
$$

where the ' indicates quantities in the imaging plane. The same line in the $3 \mathrm{D}$ coordinate system of the world ground frame is

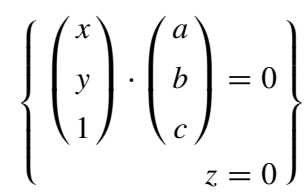

Note that we assume that $z=0$ is the ground, and that the runway is on the ground.

Any point $p=(x, y, z)^{T}$ in $3 \mathrm{D}$ in the world frame corresponds to a point $p=\left(x^{\prime}, y^{\prime}\right)^{T}$ in 2D in the imaging plane. As noted previously, the transformation is given by

$$
\begin{aligned}
\left(\begin{array}{l}
x_{\mathrm{cam}} \\
y_{\mathrm{cam}} \\
z_{\mathrm{cam}}
\end{array}\right) & =\kappa\left[R\left(\begin{array}{l}
x \\
y \\
z
\end{array}\right)+T\right] \\
\left(\begin{array}{l}
x^{\prime} \\
y^{\prime}
\end{array}\right) & =\left(\begin{array}{l}
x_{\mathrm{cam}} / z_{\mathrm{cam}} \\
y_{\mathrm{cam}} / z_{\mathrm{cam}}
\end{array}\right)
\end{aligned}
$$

where $\kappa$ is the intrinsic calibration matrix, and $(R, t)$ describe the rotation and translation between ground and camera frame.

We write Eq. (B2) in vector form as

$$
\left(\begin{array}{l}
a^{\prime} \\
b^{\prime} \\
c^{\prime}
\end{array}\right)\left(\begin{array}{l}
x \\
y \\
1
\end{array}\right)=\boldsymbol{a}_{\text {image }} \cdot\left(\begin{array}{l}
x^{\prime} \\
y^{\prime} \\
1
\end{array}\right)=0
$$


Substituting Eq. (B4) into the previous equation, we get

$$
\boldsymbol{a}_{\text {image }} \cdot\left(\begin{array}{c}
x_{\text {cam }} / z_{\text {cam }} \\
y_{\text {cam }} / z_{\text {cam }} \\
1
\end{array}\right)=0
$$

and multiplying both sides of the equation by $z_{\text {cam }}$, we arrive at

$$
\boldsymbol{a}_{\text {image }} \cdot\left(\begin{array}{c}
x_{\text {cam }} \\
y_{\text {cam }} \\
z_{\text {cam }}
\end{array}\right)=0
$$

By substituting Eq. (B3) into Eq. (B7), we arrive at

$$
\begin{gathered}
\boldsymbol{a}_{\text {image }}^{T}\left[\kappa\left(R\left(\begin{array}{l}
x \\
y \\
0
\end{array}\right)+t\right)\right]=0 \\
\left(\boldsymbol{a}_{\text {image }}^{T} \kappa R\right)\left(\begin{array}{l}
x \\
y \\
0
\end{array}\right)+\left(\boldsymbol{a}_{\text {image }}^{T} \kappa t\right)=0
\end{gathered}
$$

We can rewrite the previous expression as

$$
\left[\begin{array}{ll}
\boldsymbol{a}_{\text {image }}^{T} \kappa R \operatorname{id}_{3 \times 2} & \boldsymbol{a}_{\text {image }}^{T} \kappa t
\end{array}\right]\left(\begin{array}{l}
x \\
y \\
1
\end{array}\right)=0
$$

where $\mathrm{id}_{3 \times 2}$ is the $3 \times 2$ matrix containing as columns unit vectors, $(1,0,0)^{T}$ and $(0,1,0)^{T}$. Note that the previous equation is now in the form of the line in 3D world coordinates, as in Eq. (B2). Therefore, we can take the left most factor to be the parameters of the line in the world coordinate system:

$$
\begin{aligned}
\boldsymbol{a}_{\mathrm{world}}^{T} & =\left[\begin{array}{ll}
\boldsymbol{a}_{\text {image }}^{T} \kappa R \operatorname{id}_{3 \times 2} & \boldsymbol{a}_{\text {image }}^{T} \kappa t
\end{array}\right] \\
& =\left[\begin{array}{ll}
\boldsymbol{a}_{\text {image }}^{T} \kappa R \text { id }_{3 \times 2} & \boldsymbol{a}_{\text {image }}^{T} \kappa t
\end{array}\right] \\
& =\boldsymbol{a}_{\text {image }}^{T}\left[\begin{array}{ll}
\kappa \text { id }_{3 \times 2} & \kappa t
\end{array}\right]
\end{aligned}
$$

Transposing both sides of the equation above and then solving for $\boldsymbol{a}_{\text {image }}$, one obtains

$$
\left(\begin{array}{l}
a^{\prime} \\
b^{\prime} \\
c^{\prime}
\end{array}\right)=E\left(\begin{array}{l}
a \\
b \\
c
\end{array}\right)
$$

where

$$
E=\left[\begin{array}{ll}
\kappa R \operatorname{Rid}_{3 \times 2} & \kappa t
\end{array}\right]^{-T}=\left(\begin{array}{lll}
e_{1} & e_{2} & e_{3}
\end{array}\right)^{-T}
$$

$$
e_{1}=\left(\begin{array}{l}
k_{1} \cdot r_{1} \\
k_{2} \cdot r_{1} \\
k_{3} \cdot r_{1}
\end{array}\right), \quad e_{2}=\left(\begin{array}{l}
k_{1} \cdot r_{2} \\
k_{2} \cdot r_{2} \\
k_{3} \cdot r_{2}
\end{array}\right), \quad e_{3}=\left(\begin{array}{c}
k_{1} \cdot t \\
k_{2} \cdot t \\
k_{3} \cdot t
\end{array}\right)
$$

This same transformation can be also used to relate both parallel lines of the runway in the image; therefore, we get

$$
\left(\begin{array}{l}
a^{\prime} \\
b^{\prime} \\
c^{\prime}
\end{array}\right)=E\left(\begin{array}{l}
a \\
b \\
c
\end{array}\right), \quad\left(\begin{array}{l}
d^{\prime} \\
e^{\prime} \\
f^{\prime}
\end{array}\right)=E\left(\begin{array}{l}
a \\
b \\
d
\end{array}\right)
$$

where $d^{\prime}, e^{\prime}, f^{\prime}$ are the parameters of the second line of the runway in the image plane. By putting both of those equations into a single equation, we can get our measurement model:

$$
\left(\begin{array}{l}
a^{\prime} \\
b^{\prime} \\
c^{\prime} \\
d^{\prime} \\
e^{\prime} \\
f^{\prime}
\end{array}\right)=C_{k}\left(\begin{array}{l}
a \\
b \\
c \\
d
\end{array}\right)
$$

where $C_{t}$ is a $6 \times 4$ matrix given by

$$
C_{t}=\left(\begin{array}{cccc}
e_{1} & e_{2} & e_{3} & 0_{(3 \times 1)} \\
e_{1} & e_{2} & 0_{(3 \times 1)} & e_{3}
\end{array}\right)
$$

This equation relates the parameters of the parallel lines in the world frame to the parameters of the two lines in the imaging plane (not necessarily parallel).

\section{Appendix C: Runway Location in the Earth Frame Given Its Image Location}

In a classical camera pinhole model, the alignment of the axes is described by the following. The camera $Z$ axis is the depth axis, where the image is formed in the $X Y$ plane, and hence the depth component is the normalization scaler to reach homogenous coordinates.

Figure 6 is an illustrative figure to the following relation that back project any point, in the image plane captured by the camera in its coordinate system, into the corresponding point in the world coordinate system. Given a $2 \mathrm{D}$ point, the $3 \mathrm{D}$ point located at the ground $z=0$ can be obtained by solving a linear system. Starting with camera pinhole model, we have

$$
\kappa\left[R\left(\begin{array}{l}
x \\
y \\
z
\end{array}\right)+t\right]=\left(\begin{array}{c}
x_{\mathrm{cam}} \\
y_{\mathrm{cam}} \\
z_{\mathrm{cam}}
\end{array}\right)
$$

Hence, the normalized 2D point can be written as

$$
\left(\begin{array}{l}
x_{\mathrm{cam}} \\
y_{\mathrm{cam}}
\end{array}\right)=z_{\mathrm{cam}}\left(\begin{array}{l}
x^{\prime} \\
y^{\prime}
\end{array}\right)
$$

Now, the system can be decomposed into two parts:

$$
\operatorname{id}_{3 \times 2}^{T} \kappa R\left(\begin{array}{c}
x \\
y \\
0
\end{array}\right)+\mathrm{id}_{3 \times 2}^{T} \kappa t=z_{\mathrm{cam}}\left(\begin{array}{c}
x^{\prime} \\
y^{\prime}
\end{array}\right)
$$




$$
z_{\mathrm{cam}}=\mathrm{id}_{3 \times 1}^{T} \kappa R\left(\begin{array}{l}
x \\
y \\
0
\end{array}\right)+\mathrm{id}_{3 \times 1}^{T} \kappa t
$$

where $\mathrm{id}_{3 \times 2}$ is the $3 \times 2$ matrix containing as columns unit vectors, $(1,0,0)^{T}$ and $(0,1,0)^{T}$, and $\operatorname{id}_{3 \times 1}$ is the $3 \times 2$ matrix containing as column unit vector, $(0,0,1)^{T}$.

Combining terms and rearranging:

$$
\operatorname{id}_{3 \times 2}^{T} \kappa R\left(\begin{array}{c}
x \\
y \\
0
\end{array}\right)-\left(\begin{array}{c}
\left(\operatorname{id}_{3 \times 1}^{T} \kappa R\right) \cdot x^{\prime} \\
\left(\operatorname{id}_{3 \times 1}^{T} \kappa R\right) \cdot y^{\prime}
\end{array}\right)\left(\begin{array}{l}
x \\
y \\
0
\end{array}\right)=-\operatorname{id}_{3 \times 2}^{T} \kappa t+\operatorname{id}_{3 \times 1}^{T} \kappa t\left(\begin{array}{l}
x^{\prime} \\
y^{\prime}
\end{array}\right)
$$

Hence, solving the system $M P=b$ would give the $3 \mathrm{D}$ point, where $M$ and $b$ are given by

$$
\begin{gathered}
M=\operatorname{id}_{2 \times 2} \kappa R-\left(\begin{array}{c}
x^{\prime} \\
y^{\prime}
\end{array}\right) \operatorname{id}_{2 \times 1}^{T} \kappa R \\
b=-\operatorname{id}_{3 \times 2} \kappa t+\operatorname{id}_{3 \times 1}^{T} \kappa t\left(\begin{array}{c}
x^{\prime} \\
y^{\prime}
\end{array}\right)
\end{gathered}
$$

\section{Acknowledgment}

We gratefully acknowledge the Aerospace Division of King Abdulaziz City for Science and Technology (KACST) for supporting us with experimental flight videos generated by the SAKER 4 UAV.

\section{References}

[1] Kontitsis, M., Valavanis, K. P., and Tsourveloudis, N., "A UAV Vision System for Airborne Surveillance," 2004 IEEE International Conference on Robotics and Automation, 2004. Proceedings. ICRA'04, Vol. 1, IEEE, 2004, pp. 77-83. doi:10.1109/ROBOT.2004.1307132

[2] Medioni, G., Cohen, I., Brémond, F., Hongeng, S., and Nevatia, R., IEEE Transactions on Pattern Analysis and Machine Intelligence, Vol. 23, No. 8, 2001, pp. 873-889. doi: $10.1109 / 34.946990$

[3] Todorovic, S., and Nechyba, M. C., "Intelligent Missions for MAVs: Visual Contexts for Control, Tracking and Recognition," 2004 IEEE International Conference on Robotics and Automation, 2004. Proceedings. ICRA'04, Vol. 2, IEEE, 2004, pp. 1640-1645. doi:10.1109/ROBOT.2004.1308059

[4] Lai, A. H. S., and Yung, N. H. C., "Lane Detection by Orientation and Length Discrimination," IEEE Transactions on Systems, Man, and Cybernetics, Part B: Cybernetics, Vol. 30, No. 4, 2000, pp. 539-548. doi: $10.1109 / 3477.865171$

[5] Tang, Y.-L., and Kasturi, R., "Runway Detection in an Image Sequence," IS\&T/SPIE's Symposium on Electronic Imaging: Science \& Technology, International Society for Optics and Photonics, 1995, pp. 181-190.

doi: $10.1117 / 12.205479$

[6] Dusha, D., Boles, W. W., and Walker, R., "Fixed-Wing Attitude Estimation Using Computer Vision Based Horizon Detection," 2007.

[7] Meng, D., Yun-feng, C., and Lin, G., "A Method to Recognize and Track Runway in the Image Sequences Based on Template Matching," 1st International Symposium on Systems and Control in Aerospace and Astronautics, 2006. ISSCAA 2006, IEEE, 2006, p. 4. doi:10.1109/ISSCAA.2006.1627585

[8] Gui, Y., Guo, P., Zhang, H., Lei, Z., Zhou, X., Du, J., and Yu, Q., "Airborne Vision-Based Navigation Method for UAVAccuracy Landing Using Infrared Lamps," Journal of Intelligent \& Robotic Systems, Vol. 72, No. 2, 2013, pp. 197-218. doi:10.1007/s10846-013-9819-5

[9] Phueakjeen, W., Jindapetch, N., Kuburat, L., and Suvanvorn, N., "A Study of the Edge Detection for Road Lane," 2011 8th International Conference on Electrical Engineering/Electronics, Computer, Telecommunications and Information Technology (ECTI-CON), IEEE, 2011, pp. 995-998. doi:10.1109/ECTICON.2011.5948010

[10] Wang, Y., Teoh, E. K., and Shen, D., "Lane Detection and Tracking Using B-Snake," Image and Vision Computing, Vol. 22, No. 4, 2004, pp. 269-280. doi:10.1016/j.imavis.2003.10.003

[11] Liu, W., and Li, S., "An Effective Lane Detection Algorithm for Structured Road in Urban," Intelligent Science and Intelligent Data Engineering, Springer, 2013, pp. 759-767. doi:10.1007/978-3-642-36669-7

[12] Saripalli, S., Montgomery, J. F., and Sukhatme, G., "Vision-Based Autonomous Landing of an Unmanned Aerial Vehicle," IEEE International Conference on Robotics and Automation, 2002. Proceedings. ICRA'02, Vol. 3, IEEE, 2002, pp. 2799-2804. doi:10.1109/ROBOT.2002.1013656

[13] Lim, K. H., Seng, K. P., Ang, L.-M., and Chin, S. W., "Lane Detection and Kalman-Based Linear-Parabolic Lane Tracking," International Conference on Intelligent Human-Machine Systems and Cybernetics, 2009. IHMSC'09, Vol. 2, IEEE, 2009, pp. 351-354. doi:10.1109/IHMSC.2009.211

[14] Goldstein, T., Bresson, X., and Osher, S., "Geometric Applications of the Split Bregman Method: Segmentation and Surface Reconstruction," Journal of Scientific Computing, Vol. 45, Nos. 1-3, 2010, pp. 272-293. doi:10.1007/s10915-009-9331-Z

[15] Osher, S., Burger, M., Goldfarb, D., Xu, J., and Yin, W., "An Iterative Regularization Method for Total Variation-Based Image Restoration," Multiscale Modeling \& Simulation, Vol. 4, No. 2, 2005, pp. 460-489. doi: $10.1137 / 040605412$

[16] Laiacker, M., Kondak, K., Schwarzbach, M., and Muskardin, T., "Vision Aided Automatic Landing System for Fixed Wing UAV," 2013 IEEE/RSJ International Conference on Intelligent Robots and Systems (IROS), IEEE, 2013, pp. 2971-2976. doi:10.1109/IROS.2013.6696777

[17] Arbelaez, P., Maire, M., Fowlkes, C., and Malik, J., "Contour Detection and Hierarchical Image Segmentation," IEEE Transactions on Pattern Analysis and Machine Intelligence, Vol. 33, No. 5, 2011, pp. 898-916. doi:10.1109/TPAMI.2010.161

[18] Sofou, A., Evangelopoulos, G., and Maragos, P., "Coupled Geometric and Texture PDE-Based Segmentation," IEEE International Conference on Image Processing, 2005. ICIP 2005, Vol. 2, IEEE, 2005, p. II-650. doi:10.1109/ICIP.2005.1530139

[19] Kornprobst, P., Deriche, R., and Aubert, G., Image Sequence Restoration: A PDE Based Coupled Method for Image Restoration and Motion Segmentation, Springer, 1998. doi: $10.1007 / \mathrm{BFb} 0054764$

[20] Sofou, A., and Maragos, P., "Generalized Flooding and Multicue PDEBased Image Segmentation," IEEE Transactions on Image Processing, Vol. 17, No. 3, 2008, pp. 364-376. doi:10.1109/TIP.2007.916156

[21] Yezzi, A., Tsai, A., and Willsky, A., "A Fully Global Approach to Image Segmentation via Coupled Curve Evolution Equations," Journal of Visual Communication and Image Representation, Vol. 13, No. 1, 2002, pp. 195-216. doi:10.1006/jvci.2001.0500

[22] Boyd, S., and Vandenberghe, L., Convex Optimization, Cambridge Univ. Press, 2004

[23] Kennedy, J., "Particle Swarm Optimization," Encyclopedia of Machine Learning, Springer, 2010, pp. 760-766. doi:10.1007/978-0-387-30164-8_630

[24] Unal, G., Yezzi, A., and Krim, H., "Information-Theoretic Active Polygons for Unsupervised Texture Segmentation," International Journal of Computer Vision, Vol. 62, No. 3, 2005, pp. 199-220. doi:10.1007/s11263-005-4880-6

[25] Abu-Jbara, K., Alheadary, W., Sundaramorthi, G., and Claudel, C., "A Robust Vision-Based Runway Detection and Tracking Algorithm for Automatic UAV Landing," 2015 International Conference on Unmanned Aircraft Systems (ICUAS), IEEE, 2015, pp. 1148-1157. doi:10.1109/ICUAS.2015.7152407

[26] Zanetti, R., Majji, M., Bishop, R. H., and Mortari, D., "Norm-Constrained Kalman Filtering," Journal of Guidance, Control, and Dynamics, Vol. 32, No. 5, 2009, pp. 1458-1465. doi: $10.2514 / 1.43119$

[27] Ma, Y., Soatto, S., Kosecka, J., and Sastry, S. S., An Invitation to 3-d Vision: From Images to Geometric Models, Vol. 26, Springer Science \& Business Media, 2012.

[28] Faugeras, O. D., Luong, Q.-T., and Maybank, S. J., "Camera SelfCalibration: Theory and Experiments," Computer Vision-ECCV'92, 
Springer, 1992, pp. 321-334.

doi:10.1007/3-540-55426-2_37

[29] Bouguet, J.-Y., "Camera Calibration Toolbox for Matlab," 2004.

[30] Vedaldi, A., and Fulkerson, B., "Vlfeat: An Open and Portable Library of Computer Vision Algorithms," Proceedings of the 18th ACM International Conference on Multimedia, MM '10, ACM, New York, 2010, pp. $1469-1472$. doi: $10.1145 / 1873951.1874249$

[31] Lowe, D. G., "Object Recognition from Local Scale-Invariant Features," The Proceedings of the Seventh IEEE International Conference on Computer vision, 1999, IEEE, Vol. 2, 1999, pp. 1150-1157.

[32] Bay, H., Tuytelaars, T., and Van Gool, L., "Surf: Speeded Up Robust Features," Computer vision-ECCV 2006, 2006, pp. 404-417.
[33] Rosten, E., and Drummond, T., "Machine Learning for HighSpeed Corner Detection," Computer Vision-ECCV 2006, 2006, pp. 430-443.

[34] Abu-Jbara, K., "Runway Detection and Trackingin 3d Algorithm's Videos," https://sites.google.com/a/kaust.edu.sa/projects-kf/3Dtrackingrunway-autolanding [Accessed 2 February 2017].

[35] Irani, M., "Matlab Wrapper for Graph Cuts," http://www.wisdom. weizmann.ac.il/ bagon/matlab.html.

[36] Zhu, S. C., and Yuille, A., "Region Competition: Unifying Snakes, Region Growing, and Bayes/MDL for Multiband Image Segmentation," IEEE Transactions on Pattern Analysis and Machine Intelligence, Vol. 18, No. 9, 1996, pp. 884-900. doi: $10.1109 / 34.537343$ 


\section{Queries}

1. AU: Please check that the copyright (@) type is correct. Please note that the code will be added upon publication.

2. AU: Please add postal code next to "Texas" in the affiliation.

3. AU: Definitions of acronyms have been moved from the nomenclature into the text of the article, per AIAA style.

4. AU: The sentence "Using (12) and find the equivalent transformation that ..." is not clear. Please check.

5. AU: In "runway in the world coordinate system as in 12 ..," it is not clear if "12" indicates Ref. [12] or Eq. (12). Please correct.

6. AU: "following relation that back project any point" is not clear. Please check.

7. AU: Please provide publisher location for Refs. [1, 3, 5, 7, 9, 11-13, 16, 18, 23, 25, 28, 31].

8. AU: Please provide complete details for Refs. [6, 29].

9. AU: Please provide publisher location, and page range (or chapter number) for Refs. [19, 22, 27].

10. AU: If Refs. $[32,33]$ are published proceedings, please provide the name and location of the publisher (NOT of the conference host). If they are conference papers, please provide the paper number and the organizer's name. If they are CD-ROMs, please provide the name and location of the CD-ROM producer.

11. AU: Please provide year of publication for Refs. [34, 35]. 